\title{
Broadband infrared photometry of comet Hale-Bopp with ISOPHOT^
}

\author{
E. Grün ${ }^{1}$, M. S. Hanner ${ }^{2}$, S. B. Peschke ${ }^{1,3}$, T. Müller ${ }^{3}$, H. Boehnhardt ${ }^{4}$, T. Y. Brooke ${ }^{2}$, H. Campins ${ }^{5}$, \\ J. Crovisier ${ }^{6}$, C. Delahodde ${ }^{4}$, I. Heinrichsen ${ }^{3}$, H. U. Keller ${ }^{7}$, R. F. Knacke ${ }^{8}$, H. Krüger ${ }^{1}$, P. Lamy ${ }^{9}$, \\ Ch. Leinert ${ }^{10}$, D. Lemke ${ }^{10}$, C. M. Lisse ${ }^{11}$, M. Müller ${ }^{1,12}$, D. J. Osip ${ }^{12}$, M. Solc ${ }^{13}$, M. Stickel ${ }^{10}$, M. Sykes ${ }^{5}$, \\ V. Vanysek ${ }^{13, \dagger}$, and J. Zarnecki ${ }^{14}$
}

1 Max-Planck-Institut für Kernphysik, Heidelberg, Germany

2 Jet Propulsion Laboratory, California Institute of Technology, Pasadena CA, USA

3 ISO Data Centre, ESA, Villafranca, Spain

4 European Southern Observatory, Santiago, Chile

5 University of Arizona, Tucson AZ, USA

${ }^{6}$ Observatoire de Paris, Meudon, France

7 Max-Planck-Institut für Aeronomie, Katlenburg-Lindau, Germany

8 Penn State University, Erie PA, USA

${ }^{9}$ Laboratoire d'astrophysique spatiale, Marseille, France

10 Max-Planck-Institut für Astronomie, Heidelberg, Germany

11 Space Telescope Science Institute, Baltimore MD, USA

12 Massachusetts Institute of Technology, Cambridge MA, USA

13 Charles University, Prague, Czech Republic

14 University of Kent, Canterbury, UK

Received 25 April 2000 / Accepted 6 August 2001

\begin{abstract}
Comet Hale-Bopp was observed five times with ISOPHOT, the photometer on board ESA's Infrared Space Observatory (ISO) between 4.6 and 2.8 AU. Each time, broadband photometry was performed using 4 different detectors, 5 apertures and 10 filters covering the range between 3.6 and $170 \mu \mathrm{m}$. Background observations were performed with identical instrument settings at the same positions on the sky several days after the comet observations. The observation strategy and the data reduction steps are described in some detail, including the techniques to correct for variable detector responsivity. The resulting inband power values of the Hale-Bopp observations and their uncertainties are given. The mean uncertainty is $25 \%$. The final fluxes were computed, taking into account the zodiacal background, possible offset of the comet's position from the center of the aperture, the brightness distribution within the coma, and the spectral energy distribution of the comet's emission. Strong thermal emission from a broad size distribution of dust particles was detected in all of the data sets, even at $r=4.6-4.9$ AU pre-perihelion and 3.9 AU post-perihelion; the total thermal energy varied as $r^{-3}$. The 7.3-12.8 $\mu \mathrm{m}$ color temperature was $\sim 1.5$ times the blackbody temperature, higher than that observed in any other comet. Silicate features at 10 and $25 \mu \mathrm{m}$ were prominent in all 5 data sets, the largest heliocentric distances that silicate emission has been detected in a comet. The presence of crystalline water ice grains is suggested from the $60 \mu \mathrm{m}$ excess emission at 4.6-4.9 AU, consistent with the observed $Q_{\mathrm{OH}}$ if the icy grains were slightly warmer than an equilibrium blackbody. The average albedo of the dust is higher than that of comet $\mathrm{P} / \mathrm{Halley}$, but lower than other albedo measurements for Hale-Bopp nearer perihelion. There is no evidence for a component of cold, bright icy grains enhancing the scattered light at 4.6 AU. Simple models for a mixture of silicate and absorbing grains were fit to the ISO spectra and photometry at $2.8 \mathrm{AU}$. The observed flux at $\lambda>100 \mu \mathrm{m}$ requires a size distribution in which most of the mass is concentrated in large particles. Dust production rates of order $1.5 \times 10^{5} \mathrm{~kg} \mathrm{~s}^{-1}$ at $2.8 \mathrm{AU}$ and $3 \times 10^{4} \mathrm{~kg} \mathrm{~s}^{-1}$ at $4.6 \mathrm{AU}$ have been found. They correspond to dust to gas mass ratios of 6 to 10 .
\end{abstract}

Key words. comets: individual: C/1995 O1 (Hale-Bopp) - infrared: solar system

Send offprint requests to: E. Grün,

e-mail: eberhard.gruen@mpi-hd.mpg.de

* With supporting observations by ISOCAM, SWS, LWS, and ground-based observations at the European Southern

Observatory at La Silla in Chile.

$\dagger$ Deceased. 


\section{Introduction}

Comet C/1995 O1 (Hale-Bopp) was discovered in July 1995 at a heliocentric distance of $7.15 \mathrm{AU}$ as a faint, extended, moving 10th magnitude object in the constellation Sagittarius. It soon became clear from orbital calculations that this comet had the potential of being exceptionally bright. The discovery at large heliocentric distance offered the possibility to study the coma evolution over a large arc of the orbit. The anticipated brightness and expectations of outstanding scientific results convinced the European Space Agency (ESA) to approve comet Hale-Bopp as an exceptional target of opportunity for its Infrared Space Observatory (ISO, Kessler et al. 1996).

The comet lived up to these early predictions and was extensively observed from the ground and from Earth orbit. Hale-Bopp's perihelion passage occurred on 1 April 1997. The water production rate near perihelion was $\sim 10^{31} \mathrm{~mol} / \mathrm{s}$ (Colom et al. 1999; Weaver et al. 1999). The dust area $\times$ albedo product was two orders of magnitude higher than that of comet Halley at comparable heliocentric distances (Schleicher et al. 1997). Complex patterns of jets and halos were visible in the coma. The exceptional activity was apparently due to the comet's large nucleus (40-70 km diameter; Weaver \& Lamy 1999; Lamy et al. 1999; Sekanina 1999; Fernandez et al. 1999).

The strong activity at large heliocentric distance was driven by $\mathrm{CO}$. The measured $\mathrm{CO}$ production rate at 6.6 AU was $\sim 2 \times 10^{28}$ molecules $\mathrm{s}^{-1}$ (Biver et al. 1996; Jewitt et al. 1996). Visible dust jets indicated episodic outbursts (e.g. Sekanina 1996). Judging from the brightness of the dust coma, the dust mass loading was extremely high. Between 4 and $3 \mathrm{AU}$, the water production rate rose steeply, perhaps fed at least partly by sublimation of icy grains in the coma. (Near-infrared absorption features of water ice were detected at $r=7$ AU; Davies et al. 1997.) At $r<3 \mathrm{AU}$, water dominated the gas production (Biver et al. 1997; Weaver et al. 1997).

Comet Hale-Bopp was observable from ISO in three windows: 23 March to 28 April $1996(r \sim 4.9-4.6$ AU), 25 August to 13 October $1996(r \sim 2.9-2.8 \mathrm{AU})$, and again from late December 1997 to April 1998 at $r \geq$ 3.9 AU post-perihelion. Thus, the ISO observations sampled both the $\mathrm{CO}$-driven and $\mathrm{H}_{2} \mathrm{O}$-driven regimes. The spectroscopic results were reported by Crovisier et al. (1997a, 1997b, 1999). These results include the discovery of strong emission features from crystalline olivine at wavelengths $10-33 \mu \mathrm{m}$. ISOCAM results have been described by Lamy et al. (1999) and Jorda et al. (2000a). In this paper we present the photometric measurements taken with ISOPHOT, spanning the wavelength range from 3.6 to $170 \mu \mathrm{m}$.

Infrared photometry allows an estimate of the amount of dust in the coma over time and the rate of mass loss from the nucleus. The spectral energy distribution over a broad range in wavelength provides information on the size and composition of the dust grains, via their wavelength-dependent emissivities. While the 1-20 $\mu \mathrm{m}$ region is accessible from the ground in atmospheric windows, only space-borne instruments can sample the full wavelength range, extending beyond $100 \mu \mathrm{m}$.

The ISOPHOT observations focused on the following scientific objectives:

- measuring the dust thermal energy distribution over a large span of heliocentric distances;

- investigating the transition from volatile gas $(\mathrm{CO})$ to water-dominated coma;

- investigating any changes of dust properties (composition, temperatures, size distribution) as a function of heliocentric distance.

The aim of this paper is to describe the observations and the initial data reduction scheme, especially the signal calibration, in enough detail in order to understand the uncertainties of the measurement results. The paper is organized in the following way: the ISO and ground-based observations are described in Sect. 2. By improved initial data processing, physical quantities (inband power) and their uncertainties are derived from raw measurement data taking detector calibration into account (Sect. 3 and Appendices). Astronomical data reduction and numerical processing allowed us to calculate flux values and to obtain the spectral energy distribution by taking into account the geometry and color of the object observed (Sect. 3.1). In Sect. 4 we discuss the dust properties inferred from the infrared photometry.

\section{Observations}

\subsection{ISOPHOT photometry}

Photometric observations of comet Hale-Bopp were performed with the ISOPHOT instrument (Lemke et al. 1996). ISO viewing constraints together with the comet's orbital geometry limited the visibility of HaleBopp for ISO. Observations were only allowed in the solar elongation range of $60^{\circ}-120^{\circ}$. In addition, the Earth had to be at least $24^{\circ}$ away from the telescope axis. Further pointing constraints were imposed by the Moon and Jupiter.

Within the visibility period of Hale-Bopp, the lowest possible background flux (absolute level, no point sources) had to be found at which the observations were to be performed. The search for the lowest background along the arc of Hale-Bopp's orbit was done in two sequential steps (Peschke 1997). First, a raw selection based on the IRAS all-sky survey (ISSA) plates was performed. "Dark regions" at 12, 25, 60 and $100 \mu \mathrm{m}$ along the cometary track on the ISSA plates were selected. Second, the selected regions were examined with IRSKY (http:// www. ipac. caltech. edu/ ipac/ services/ irsky.html) to assure that no known point source was within $5^{\prime}$ of the cometary track, and no prominent (cirrus) structures were visible on the $100 \mu \mathrm{m}$ ISSA plates. Also background 
Table 1. Summary log of Hale-Bopp observations with ISOPHOT. For each observation (master observation, M and shadow observations, S) observing date, time period, the heliocentric distance $r$ and the geocentric distance, $\Delta$, of the comet are listed as well as the filters that were used for the observations. The apertures for the multi-filter photometry were selected such that at least $90 \%$ of the incident radiation from a point source reached the detector.

\begin{tabular}{ccccccl}
\hline Obs. & Master/shadow & \multicolumn{1}{c}{ Date } & Period (UT) & $r[\mathrm{AU}]$ & $\Delta[\mathrm{AU}]$ & \multicolumn{1}{l}{ Filters $[\mu \mathrm{m}]$} \\
\hline 0 & $\mathrm{M}$ & 25-Mar.-96 & $17: 31: 25-18: 17: 03$ & 4.92 & 5.15 & $3.6,7.3,10,12.8,15,60,100,170$ \\
0 & $\mathrm{~S}$ & 30-Mar.-96 & $17: 27: 32-18: 00: 22$ & & & $3.6,7.3,10,12.8,15,60,100,170$ \\
1 & $\mathrm{M}$ & 27-Apr.-96 & $15: 38: 28-16: 21: 58$ & 4.58 & 4.25 & $7.3,10,12.8,15,25,60,100,170$ \\
1 & $\mathrm{~S}$ & 5-May-96 & $6: 09: 11-6: 42: 37$ & & & $7.3,10,12.8,15,25,60,100,170$ \\
2 & $\mathrm{M}$ & 27-Sep.-96 & $3: 50: 31-5: 17: 19$ & 2.93 & 2.97 & $3.6,7.3,10,12.8,15,60,100,170$ \\
2 & $\mathrm{~S}$ & 6-Sep.-96 & $16: 29: 54-16: 59: 49$ & & & $3.6,7.3,10,12.8,15,60,100,170$ \\
3 & $\mathrm{M}$ & 7-Oct.-96 & $5: 26: 48-6: 13: 24^{1}$ & 2.82 & \multirow{2}{*}{3.01} & $3.6,7.3,10,12.8,15,25,60,100,170$ \\
3 & $\mathrm{~S}$ & 10-Oct.-96 & $15: 08: 18-17: 04: 14$ & & & $3.6,7.3,10,12.8,15,25,60,100,170$ \\
4 & $\mathrm{M}$ & 30-Dec.-97 & $1: 29: 39-2: 31: 41$ & 3.90 & \multirow{2}{*}{3.74} & $3.6,7.3,10,11.3,12.8,15,25,60,100,170$ \\
4 & $\mathrm{~S}$ & 4-Jan.-98 & $3: 13: 05-4: 06: 35$ & & & $3.6,7.3,10,11.3,12.8,15,25,60,100,170$ \\
\hline
\end{tabular}

${ }^{1}$ : The $25 \mu \mathrm{m}$ observation was taken on 8-Oct.-96 14:00:30-14:04:16.

Table 2. Complementary observations used in this paper.

\begin{tabular}{rllcll}
\hline \multicolumn{1}{c}{ Date } & Period (UT) & Filter & $\lambda_{\text {eff }}[\mu \mathrm{m}]$ & Instrument & Observer/Reference \\
\hline 27-Apr.-96 & $6: 05-8: 00$ & $R$ & 0.7 & Danish 1.5-m & Rauer, Boehnhardt \\
27-Apr.-96 & $14: 24-15: 36$ & & $2.5-11.6$ & PHT-S & Crovisier et al. (1996) \\
17-Sep.-96 & $1: 30-1: 40$ & $R$ & 0.7 & ESO 2.2-m & Schulz, Tozzi \\
1-Oct.-96 & $14: 12$ & LW9 & 15.0 & ISOCAM & Jorda et al. (2000b) \\
4-Oct.-96 & $0: 00-0: 05$ & $R$ & 0.7 & Danish 1.5 m & Rauer \\
6/7-Oct.-96 & $23: 31-0: 29$ & & $43-195$ & LWS & Lellouch et al. (1998) \\
7-Oct.-96 & $0: 29-2: 53$ & & $2.4-45$ & SWS & Crovisier et al. (1997a) \\
7-Oct.-96 & $4: 05-5: 17$ & & $2.5-11.6$ & PHT-S & Crovisier et al. (1997a) \\
7-Oct.-96 & $6: 32$ & LW9 & 15.0 & ISOCAM & Jorda et al. (2000b) \\
25-Dec.-97 & $0: 14-1: 26$ & & $2.5-11.6$ & PHT-S & Crovisier et al. (1999) \\
28-Dec.-97 & $2: 10-4: 19$ & & $2.4-45$ & SWS & Crovisier et al. (1999) \\
\hline
\end{tabular}

estimates for each filter of the planned ISO observations were derived with IRSKY.

Background subtraction is crucial for photometric measurements, but standard simultaneous offset measurements (chopped observations) were dismissed because of the extended coma of Hale-Bopp. Instead, a shadow measurement is the repetition of the Hale-Bopp measurement, called "master observation". A shadow measurement was performed at the same position on the sky that was tracked in the master observation, at a time when the comet and, more importantly, the coma had moved away from that sky position. Shadow observations were performed about one week after the master observations. The zodiacal background at thermal emission wavelengths changes roughly $1 \%$ per day, from the COBE/DIRBE observations. So performing a shadow observation of the background within a few days' time leads to only a few percent systematic error. Because of an ephemeris problem, the September 1996 Hale-Bopp observation had to be repeated 4 weeks later and, therefore, these master and shadow observations are a few degrees apart.

Four different detectors of ISOPHOT (Lemke et al. 1996) were used for the observations (Table 1): detector P1, a Si:Ga-detector for observations up to $15 \mu \mathrm{m}$; detector P2, a Si:B-detector for the $25 \mu \mathrm{m}$ observations; the C100 camera, a $3 \times 3$-pixel-array of nine Ge:Ga-detectors for 60 and $100 \mu \mathrm{m}$ observations; and the C200 camera, a $2 \times 2$ array of four stressed Ge:Ga-detectors for the $170 \mu \mathrm{m}$ observations. All observations were done in single pointing, single filter absolute photometry mode (AOT P05 and P25), i.e. with a subsequent measurement of the Fine Calibration Source (FCS). Here, we do not report on the multi-aperture measurements (AOT P04) because they were not yet at the same status of data processing.

\subsection{Complementary observations}

Complementary ground-based and ISO observations are listed in Table 2. These images and spectra aid the interpretation of the ISOPHOT data. Observations with the ISO spectrometers were performed on 6-7 October, close in time to the ISOPHOT observations. The SWS spectra of Hale-Bopp have been discussed by Crovisier et al. (1997a, 1997b, 1999). They are taken in segments through rectangular apertures of size $14^{\prime \prime} \times 20^{\prime \prime}$ to $20^{\prime \prime} \times 33^{\prime \prime}$ (Sect. 4.2). The LWS spectrometer (Clegg et al. 1996) has a $100^{\prime \prime}$ diameter field of view and spectral resolution 200; the Hale-Bopp spectrum has been presented by Lellouch et al. (1998). PHT-S, the spectroscopy mode of ISOPHOT, provided spectra shortward of $11.6 \mu \mathrm{m}$ at each epoch of the photometry (Crovisier et al. 1999). 
Preliminary ISOCAM data were provided by Lamy and the Hale-Bopp Target-of-Opportunity Team.

The ground-based observations were executed at the European Southern Observatory (ESO) as part of a larger study of coma morphology (Boehnhardt et al. 1999). We have selected the best Bessel $R$ filter data taken closest in time to the ISOPHOT observations. The Danish 1.5-m telescope was used with DFOSC on 27 April and 4 October and the MPI/ESO 2.2-m telescope was used with EFOSC2 on 17 September 1996. DFOSC and EFOSC2 are focal reducer type instruments for imaging and spectroscopy in the visible wavelength range, mounted to the Cassegrain focus of the respective telescopes. The image scale of the $2048 \times 2048$ pixel CCD is $0.41^{\prime \prime} /$ pixel for DFOSC and $0.27^{\prime \prime} /$ pixel for EFOSC2. The observing nights mentioned above had photometric sky conditions and the usual set of calibration images (bias, skyflats, 4-8 photometric standard star fields from the list of Landolt (1992) were taken. Since the coma of Comet Hale-Bopp extended over the edge of the instrument's field of view, separate sky exposures were obtained with the telescope offset from the coma center by 1-2 degrees towards the Sun. For the HaleBopp exposures, differential autoguiding with the speed of the comet was applied in order to achieve the best image quality for the moving object. The basic computer reduction of the data corrected for bias, flatfield, sky level and atmospheric extinction and achieved a photometric accuracy of the flux better than 5 percent. These images were used to determine the radial brightness gradient in the coma.

\section{Data reduction}

\subsection{Improved initial data processing}

The five ISOPHOT Hale-Bopp observation sequences reported here comprise 558 individual measurements, counting all object, shadow, and fine calibration source (FCS) measurements at up to 10 wavelengths. Some of them were multi-pixel measurements with up to 9 pixels. Each of these measurements had to undergo all initial steps of data processing described in the Appendices before they were combined into 44 Hale-Bopp and background signal values.

The initial data processing steps are:

(1) Derive the slope of the voltage ramps $[\mathrm{V} / \mathrm{s}]$ from the instrument raw data, for on- and off-target and FCS measurements (Appendix A);

(2) determine the responsivities of the detectors at the times of the observations (Appendix B);

(3) derive inband power values (Appendix $C$ ) and their uncertainties (Appendix D).

The result is shown in Table 3 .

The goal of the astronomical data processing was to derive flux values $F_{\nu}(\mathrm{Jy})$ for the coma of Hale-Bopp from inband power values. As a first step, the background (shadow) inband power values were subtracted from the Hale-Bopp values. In the second step, flux densities in the beam were calculated from the inband power values by assuming a standard spectrum $\left(\nu \cdot F_{\nu}=\right.$ constant $)$. The third step corrected for the offset between the center of the aperture during the observation and the comet position. The next correction took into account the size of the aperture used in the observation and normalized the flux to a standard aperture of $23^{\prime \prime}$ diameter. The last two corrections involved an assumed radial coma profile and the (wavelength dependent) theoretical point-spread-function, PSF, for each wavelength used. The final color correction took into account the shape of the observed spectrum versus the previously assumed standard spectrum. In the following paragraphs these corrections are described in some detail.

\subsection{Background subtraction}

Generally, the positions of the background (shadow) observations were sufficiently close (a few arc seconds) to the comet observations that the shadow inband power value could be subtracted from the comet value as planned (Table 4). Only the September 1996 observations were almost $3^{\circ}$ apart. Therefore, we took the 10 October shadow observation to correct for the background of the 27 September observation. The actual ISO observation positions were taken from the pointing information (IIPHfiles) provided with each observation. ISO-centric comet positions were calculated from comet ephemeris that were available after the observations, using the ISO-SSO tool and the ISO orbit file. By doing that, we discovered significant deviations (up to 23.8" in December 1997) between the Hale-Bopp position and the actual ISO observations. The position offsets are summarized in Table 4.

\subsection{Flux calibration}

Flux densities in the beam were calculated from the inband power values, IBP, according to

$$
F_{\nu}=\frac{\mathrm{IBP}}{C_{1}}
$$

where $C_{1}$ is a constant per detector and filter determined during instrument calibration (as contained in the PIA Cal G-files) by assuming a standard spectrum, $\nu \cdot F_{\nu}=$ const. Since the Hale-Bopp spectra deviate significantly from this power law spectrum a color correction has to be applied (see below). Note that for P1 and P2 detectors, PIA calculates the flux density of a point source by correcting for the fraction of the PSF outside the aperture. However, we are interested in the conversion factors, $C_{1}$, from inband power to flux density in the beam limited by the aperture used (Table 5).

\subsection{Radial brightness profile}

Several of the astronomical correction factors depend on the brightness distribution across the coma. Radial dust 
Table 3. Log of Hale-Bopp and corresponding shadow observation parameters. For each individual ISOPHOT measurement the following parameters are given: 1. Obs. (observation number and master/shadow indicator); 2. detector; 3. identifier; 4. wavelength $(\lambda[\mu \mathrm{m}])$ of filter; 5. aper (aperture size); 6. intt (total integration time); 7. response (responsivities used); 8. signal (resulting inband power value, IBP); 9. uncert. (total relative uncertainty): u.l. = upper limit; 10. type $^{1}$ (responsivity type); 11. proc. $^{2}$ (signal processing); 12. remarks ${ }^{3}$ (cf. appendices for further explanation).

\begin{tabular}{|c|c|c|c|c|c|c|c|c|c|c|c|}
\hline Obs. & detector & identifier & $\lambda[\mathrm{m}]$ & aper ["] & $\operatorname{intt}[s]$ & respons $[\mathrm{A} / \mathrm{W}]$ & signal $[\mathrm{W}]$ & uncert & type $^{1}$ & proc. $^{2}$ & remarks $^{3}$ \\
\hline$\overline{0 M}$ & $\mathrm{P} 1$ & 12901707 & 3.6 & 23 & 256 & 1.18 & $4.3 \times 10^{-16}$ & u.l. & $\mathrm{d}$ & $\mathrm{D}$ & w1 \\
\hline $0 \mathrm{M}$ & $\mathrm{P} 1$ & 12901708 & 7.3 & 23 & 32 & 0.88 & $5.2 \times 10^{-15}$ & 0.45 & c & M & r3 \\
\hline $0 \mathrm{M}$ & $\mathrm{P} 1$ & 12901709 & 10.0 & 52 & 32 & 1.21 & $2.7 \times 10^{-14}$ & 0.14 & $\mathrm{c}$ & M & r3, so \\
\hline $0 \mathrm{M}$ & $\mathrm{P} 1$ & 12901710 & 12.8 & 52 & 32 & 1.30 & $2.4 \times 10^{-14}$ & 0.14 & $\mathrm{c}$ & M & r3, so \\
\hline $0 \mathrm{M}$ & $\mathrm{P} 1$ & 12901711 & 15.0 & 52 & 32 & 1.36 & $1.8 \times 10^{-14}$ & 0.17 & $\mathrm{c}$ & M & so \\
\hline $0 \mathrm{M}$ & $\mathrm{C} 1$ & 12901704 & 60.0 & 135 & 32 & 57.57 & $6.1 \times 10^{-14}$ & 0.62 & $\mathrm{n}$ & $\mathrm{F}$ & sF, w2 \\
\hline $0 \mathrm{M}$ & $\mathrm{C} 1$ & 12901705 & 100.0 & 135 & 32 & 81.68 & $3.2 \times 10^{-14}$ & 0.48 & $\mathrm{n}$ & $\mathrm{F}$ & $\mathrm{sF}$ \\
\hline $0 \mathrm{M}$ & $\mathrm{C} 2$ & 12901706 & 170.0 & 180 & 32 & 22.09 & $2.3 \times 10^{-14}$ & 0.19 & c & M & \\
\hline OS & $\mathrm{P} 1$ & 13402419 & 3.6 & 23 & 256 & 1.18 & $2.7 \times 10^{-16}$ & u.l. & $\bar{d}$ & $\mathrm{D}$ & w1 \\
\hline OS & $\mathrm{P} 1$ & 13402420 & 7.3 & 23 & 32 & 1.90 & $9.2 \times 10^{-16}$ & 0.22 & $\mathrm{c}$ & $\mathrm{F}$ & \\
\hline OS & $\mathrm{P} 1$ & 13402421 & 10.0 & 52 & 32 & 1.64 & $5.3 \times 10^{-15}$ & 0.22 & $\mathrm{c}$ & $\mathrm{F}$ & \\
\hline OS & $\mathrm{P} 1$ & 13402422 & 12.8 & 52 & 32 & 2.04 & $5.7 \times 10^{-15}$ & 0.31 & c & $\mathrm{F}$ & \\
\hline OS & $\mathrm{P} 1$ & 13402423 & 15.0 & 52 & 32 & 2.30 & $3.1 \times 10^{-15}$ & 0.14 & $\mathrm{c}$ & $\mathrm{F}$ & \\
\hline OS & $\mathrm{P} 2$ & 13402424 & 25.0 & 99 & 32 & 0.71 & $7.9 \times 10^{-14}$ & 0.16 & $\mathrm{c}$ & M & \\
\hline OS & $\mathrm{C} 1$ & 13402416 & 60.0 & 135 & 32 & 96.23 & $1.1 \times 10^{-14}$ & 0.18 & $\mathrm{c}$ & M & $\mathrm{r} 3, \mathrm{sF}$ \\
\hline OS & $\mathrm{C} 1$ & 13402417 & 100.0 & 135 & 32 & 94.77 & $9.9 \times 10^{-15}$ & 0.18 & $\mathrm{c}$ & M & $\mathrm{sF}$ \\
\hline OS & $\mathrm{C} 2$ & 13402418 & 170.0 & 180 & 32 & 20.95 & $1.7 \times 10^{-14}$ & 0.08 & c & M & \\
\hline $1 \mathrm{M}$ & $\mathrm{P} 1$ & 16201331 & 3.6 & 23 & 256 & 1.18 & $1.7 \times 10^{-16}$ & u.l. & $\mathrm{d}$ & $\mathrm{D}$ & $\mathrm{w} 1$ \\
\hline $1 \mathrm{M}$ & $\mathrm{P} 1$ & 16201332 & 7.3 & 23 & 32 & 0.88 & $4.0 \times 10^{-15}$ & 0.50 & c & M & r3 \\
\hline $1 \mathrm{M}$ & $\mathrm{P} 1$ & 16201333 & 10.0 & 52 & 32 & 1.24 & $3.1 \times 10^{-14}$ & 0.14 & $\mathrm{c}$ & M & r3, so \\
\hline $1 \mathrm{M}$ & $\mathrm{P} 1$ & 16201334 & 12.8 & 52 & 32 & 1.26 & $2.9 \times 10^{-14}$ & 0.14 & c & M & r3, so \\
\hline $1 \mathrm{M}$ & $\mathrm{P} 1$ & 16201335 & 15.0 & 52 & 32 & 1.37 & $2.0 \times 10^{-14}$ & 0.14 & c & M & so \\
\hline $1 \mathrm{M}$ & $\mathrm{P} 2$ & 16201336 & 25.0 & 99 & 32 & 0.47 & $5.0 \times 10^{-13}$ & 0.10 & c & M & r3, so \\
\hline $1 \mathrm{M}$ & $\mathrm{C} 1$ & 16201328 & 60.0 & 135 & 32 & 61.23 & $5.8 \times 10^{-14}$ & 0.24 & $\mathrm{n}$ & $\mathrm{F}$ & $\mathrm{sF}$ \\
\hline $1 \mathrm{M}$ & $\mathrm{C} 1$ & 16201329 & 100.0 & 135 & 32 & 91.47 & $1.8 \times 10^{-14}$ & 0.02 & $\mathrm{n}$ & $\mathrm{F}$ & $\mathrm{sF}$ \\
\hline $1 \mathrm{M}$ & $\mathrm{C} 2$ & 16201330 & 170.0 & 180 & 32 & 22.66 & $2.2 \times 10^{-14}$ & 0.17 & c & M & \\
\hline $1 \mathrm{~S}$ & $\mathrm{P} 1$ & 17000843 & 3.6 & 23 & 256 & 1.17 & $2.7 \times 10^{-17}$ & u.l. & $\mathrm{d}$ & $\mathrm{D}$ & w1 \\
\hline $1 \mathrm{~S}$ & $\mathrm{P} 1$ & 17000844 & 7.3 & 23 & 32 & 1.17 & $7.1 \times 10^{-16}$ & 0.44 & d & $\mathrm{F}$ & w1 \\
\hline $1 \mathrm{~S}$ & $\mathrm{P} 1$ & 17000845 & 10.0 & 52 & 32 & 1.60 & $2.6 \times 10^{-15}$ & 0.14 & $\mathrm{c}$ & $\mathrm{F}$ & \\
\hline $1 \mathrm{~S}$ & $\mathrm{P} 1$ & 17000846 & 12.8 & 52 & 32 & 2.23 & $3.0 \times 10^{-15}$ & 0.28 & $\mathrm{c}$ & M & \\
\hline $1 \mathrm{~S}$ & $\mathrm{P} 1$ & 17000847 & 15.0 & 52 & 32 & 2.16 & $1.7 \times 10^{-15}$ & 0.14 & c & $\mathrm{F}$ & \\
\hline $1 \mathrm{~S}$ & $\mathrm{P} 2$ & 17000848 & 25.0 & 99 & 32 & 0.64 & $4.4 \times 10^{-14}$ & 0.16 & c & M & \\
\hline $1 \mathrm{~S}$ & $\mathrm{C} 1$ & 17000840 & 60.0 & 135 & 32 & 61.75 & $5.5 \times 10^{-15}$ & 0.33 & $\mathrm{c}$ & M & $\mathrm{r} 3, \mathrm{sF}$ \\
\hline $1 \mathrm{~S}$ & $\mathrm{C} 1$ & 17000841 & 100.0 & 135 & 32 & 56.74 & $6.7 \times 10^{-15}$ & 0.45 & $\mathrm{c}$ & M & $\mathrm{r} 3, \mathrm{sF}$ \\
\hline $1 \mathrm{~S}$ & $\mathrm{C} 2$ & 17000842 & 170.0 & 180 & 32 & 21.05 & $1.4 \times 10^{-14}$ & 0.10 & $\mathrm{c}$ & M & $\mathrm{sF}$ \\
\hline $2 \mathrm{M}$ & $\mathrm{P} 1$ & 31501055 & 3.6 & 23 & 256 & 1.18 & $2.3 \times 10^{-15}$ & 0.34 & $\mathrm{~d}$ & $\mathrm{~F}$ & w1 \\
\hline $2 \mathrm{M}$ & $\mathrm{P} 1$ & 31501556 & 7.3 & 23 & 32 & 1.39 & $8.7 \times 10^{-14}$ & 0.31 & c & M & r3, so \\
\hline $2 \mathrm{M}$ & $\mathrm{P} 1$ & 31501657 & 10.0 & 52 & 32 & 1.84 & $2.5 \times 10^{-13}$ & 0.39 & $\mathrm{c}$ & M & $\mathrm{r} 3, \mathrm{sF}, \mathrm{so}$ \\
\hline $2 \mathrm{M}$ & $\mathrm{P} 1$ & 31501758 & 12.8 & 52 & 32 & 1.51 & $1.9 \times 10^{-13}$ & 0.28 & $\mathrm{c}$ & M & r3, so \\
\hline $2 \mathrm{M}$ & $\mathrm{P} 1$ & 31501859 & 15.0 & 52 & 32 & 1.17 & $2.4 \times 10^{-13}$ & 0.25 & c & M & $\mathrm{r} 3, \mathrm{sF}, \mathrm{so}$ \\
\hline $2 \mathrm{M}$ & $\mathrm{C} 1$ & 31501352 & 60.0 & 135 & 32 & 76.61 & $1.3 \times 10^{-13}$ & 0.14 & c & M & r3 \\
\hline $2 \mathrm{M}$ & $\mathrm{C} 1$ & 31501453 & 100.0 & 135 & 32 & 75.75 & $5.4 \times 10^{-14}$ & 0.14 & c & M & r3 \\
\hline $2 \mathrm{M}$ & $\mathrm{C} 2$ & 31501954 & 170.0 & 180 & 32 & 21.89 & $6.2 \times 10^{-14}$ & 0.10 & c & M & \\
\hline $2 \mathrm{~S}$ & $\mathrm{P} 1$ & 29500767 & 3.6 & 23 & 256 & 1.16 & $1.5 \times 10^{-16}$ & u.l. & $\bar{d}$ & $\mathrm{D}$ & w1 \\
\hline $2 \mathrm{~S}$ & $\mathrm{P} 1$ & 29500868 & 7.3 & 23 & 32 & 1.16 & $8.8 \times 10^{-16}$ & 0.40 & d & $\mathrm{F}$ & w1 \\
\hline $2 \mathrm{~S}$ & $\mathrm{P} 1$ & 29500969 & 10.0 & 52 & 32 & 1.69 & $3.3 \times 10^{-15}$ & 0.20 & c & M & \\
\hline $2 \mathrm{~S}$ & $\mathrm{P} 1$ & 29501070 & 12.8 & 52 & 32 & 2.20 & $3.1 \times 10^{-15}$ & 0.27 & $\mathrm{c}$ & M & \\
\hline $2 \mathrm{~S}$ & $\mathrm{P} 1$ & 29501171 & 15.0 & 52 & 32 & 2.03 & $1.9 \times 10^{-15}$ & 0.14 & $\mathrm{c}$ & $\mathrm{F}$ & \\
\hline $2 \mathrm{~S}$ & $\mathrm{C} 1$ & 29500464 & 60.0 & 135 & 32 & 56.30 & $6.3 \times 10^{-15}$ & 0.62 & $\mathrm{c}$ & M & \\
\hline $2 \mathrm{~S}$ & $\mathrm{C} 1$ & 29500565 & 100.0 & 135 & 32 & 48.17 & $1.6 \times 10^{-14}$ & 0.91 & $\mathrm{c}$ & M & \\
\hline $2 \mathrm{~S}$ & $\mathrm{C} 2$ & 29500666 & 170.0 & 180 & 32 & 25.99 & $5.8 \times 10^{-14}$ & 0.21 & c & M & \\
\hline
\end{tabular}

1: $\mathrm{d}=$ default, $\mathrm{c}=$ corrected actual, $\mathrm{n}=$ nominal;

2: $\mathrm{M}=$ myriad, $\mathrm{F}=$ first order, $\mathrm{D}=$ dark current;

3: signal-FCS ratio: $\mathrm{r} 3=r_{\mathrm{SCP}}>3$, saturation indicators: $\mathrm{sF}$ for FCS and so for object; FCS warnings: w1 $=$ FCS heating power below calibrated range, w $2=$ FCS heating power above calibrated range. 
Table 3. continued.

\begin{tabular}{|c|c|c|c|c|c|c|c|c|c|c|c|}
\hline Obs. & detector & identifier & $\lambda[\mu \mathrm{m}]$ & aper $\left[{ }^{\prime \prime}\right]$ & $\operatorname{intt}[\mathrm{s}]$ & respons $[\mathrm{A} / \mathrm{W}]$ & signal $[W]$ & uncert & type $^{1}$ & proc. $^{2}$ & remarks ${ }^{3}$ \\
\hline $3 \mathrm{M}$ & $\mathrm{P} 1$ & 32501379 & 3.6 & 23 & 256 & 1.18 & $1.8 \times 10^{-15}$ & 0.36 & d & $\mathrm{F}$ & $\mathrm{w} 1$ \\
\hline $3 \mathrm{M}$ & $\mathrm{P} 1$ & 32501380 & 7.3 & 23 & 32 & 1.11 & $9.0 \times 10^{-14}$ & 0.14 & c & M & \\
\hline $3 \mathrm{M}$ & $\mathrm{P} 1$ & 32501381 & 10.0 & 52 & 32 & 1.07 & $2.9 \times 10^{-13}$ & 0.10 & $\mathrm{c}$ & $\mathrm{M}$ & r3 \\
\hline $3 \mathrm{M}$ & $\mathrm{P} 1$ & 32501382 & 12.8 & 52 & 32 & 1.05 & $2.0 \times 10^{-13}$ & 0.10 & $\mathrm{c}$ & M & \\
\hline $3 \mathrm{M}$ & $\mathrm{P} 1$ & 32501383 & 15.0 & 52 & 32 & 1.19 & $2.0 \times 10^{-13}$ & 0.21 & c & M & \\
\hline $3 \mathrm{M}$ & $\mathrm{P} 2$ & 32700284 & 25.0 & 99 & 32 & 0.32 & $4.6 \times 10^{-12}$ & 0.20 & $\mathrm{c}$ & M & r3, so \\
\hline $3 \mathrm{M}$ & $\mathrm{C} 1$ & 32501376 & 60.0 & 135 & 32 & 79.61 & $1.2 \times 10^{-13}$ & 0.14 & $\mathrm{c}$ & M & r3 \\
\hline $3 \mathrm{M}$ & $\mathrm{C} 1$ & 32501377 & 100.0 & 135 & 32 & 85.40 & $4.8 \times 10^{-14}$ & 0.14 & $\mathrm{c}$ & M & r3 \\
\hline $3 \mathrm{M}$ & $\mathrm{C} 2$ & 32501378 & 170.0 & 180 & 32 & 22.83 & $6.2 \times 10^{-14}$ & 0.12 & $\mathrm{c}$ & $\mathrm{M}$ & \\
\hline $3 \mathrm{~S}$ & $\mathrm{P} 1$ & 32900591 & 3.6 & 23 & 256 & 1.16 & $2.3 \times 10^{-17}$ & u.l. & $\bar{d}$ & $\mathrm{D}$ & $\mathrm{w} 1$ \\
\hline $3 \mathrm{~S}$ & $\mathrm{P} 1$ & 32900592 & 7.3 & 23 & 32 & 1.16 & $1.1 \times 10^{-15}$ & 0.35 & d & $\mathrm{F}$ & $\mathrm{w} 1$ \\
\hline $3 \mathrm{~S}$ & $\mathrm{P} 1$ & 32900593 & 10.0 & 52 & 32 & 1.37 & $4.3 \times 10^{-15}$ & 0.14 & c & $\mathrm{F}$ & \\
\hline $3 S$ & $\mathrm{P} 1$ & 32900594 & 12.8 & 52 & 32 & 1.90 & $4.3 \times 10^{-15}$ & 0.19 & $\mathrm{c}$ & M & \\
\hline $3 S$ & $\mathrm{P} 1$ & 32900595 & 15.0 & 52 & 32 & 2.05 & $2.2 \times 10^{-15}$ & 0.14 & c & $\mathrm{F}$ & \\
\hline $3 S$ & $\mathrm{P} 2$ & 32900796 & 25.0 & 99 & 32 & 0.71 & $5.2 \times 10^{-14}$ & 0.16 & c & M & \\
\hline $3 \mathrm{~S}$ & $\mathrm{C} 1$ & 32900588 & 60.0 & 135 & 32 & 62.47 & $5.9 \times 10^{-15}$ & 0.53 & $\mathrm{c}$ & M & \\
\hline $3 S$ & $\mathrm{C} 1$ & 32900589 & 100.0 & 135 & 32 & 53.34 & $1.1 \times 10^{-14}$ & 0.86 & $\mathrm{c}$ & M & \\
\hline $3 \mathrm{~S}$ & $\mathrm{C} 2$ & 32900590 & 170.0 & 180 & 32 & 25.78 & $3.9 \times 10^{-14}$ & 0.19 & $\mathrm{c}$ & M & \\
\hline $4 \mathrm{M}$ & $\mathrm{P} 1$ & 77501125 & 3.6 & 23 & 256 & 1.18 & $2.9 \times 10^{-16}$ & u.l. & $\mathrm{d}$ & $\mathrm{D}$ & $\mathrm{w} 1$ \\
\hline $4 \mathrm{M}$ & $\mathrm{P} 1$ & 77501107 & 7.3 & 23 & 256 & 1.01 & $8.5 \times 10^{-15}$ & 0.16 & $\mathrm{c}$ & $\mathrm{F}$ & \\
\hline $4 \mathrm{M}$ & $\mathrm{P} 1$ & 77501108 & 10.0 & 52 & 64 & 0.92 & $6.4 \times 10^{-14}$ & 0.23 & $\mathrm{c}$ & M & r3 \\
\hline $4 \mathrm{M}$ & $\mathrm{P} 1$ & 77501109 & 11.3 & 52 & 64 & 0.86 & $8.4 \times 10^{-14}$ & 0.38 & $\mathrm{c}$ & M & \\
\hline $4 \mathrm{M}$ & $\mathrm{P} 1$ & 77501110 & 12.8 & 52 & 64 & 1.05 & $4.3 \times 10^{-14}$ & 0.14 & c & M & \\
\hline $4 \mathrm{M}$ & $\mathrm{P} 1$ & 77501111 & 15.0 & 52 & 64 & 1.33 & $3.7 \times 10^{-14}$ & 0.14 & $\mathrm{c}$ & M & $\mathrm{sF}$ \\
\hline $4 \mathrm{M}$ & P2 & 77501112 & 25.0 & 99 & 64 & 0.39 & $1.0 \times 10^{-12}$ & 0.24 & $\mathrm{c}$ & M & r3 \\
\hline $4 \mathrm{M}$ & $\mathrm{C} 1$ & 77501104 & 60.0 & 135 & 64 & 63.23 & $5.4 \times 10^{-14}$ & 0.17 & $\mathrm{c}$ & M & r3 \\
\hline $4 \mathrm{M}$ & $\mathrm{C} 1$ & 77501105 & 100.0 & 135 & 64 & 58.65 & $2.7 \times 10^{-14}$ & 0.35 & c & M & r3 \\
\hline $4 \mathrm{M}$ & $\mathrm{C} 2$ & 77501106 & 170.0 & 180 & 64 & 23.10 & $2.4 \times 10^{-14}$ & 0.08 & c & M & \\
\hline $4 \mathrm{~S}$ & $\mathrm{P} 1$ & 78001226 & 3.6 & 23 & 256 & 1.18 & $2.9 \times 10^{-18}$ & u.l. & $\bar{d}$ & D & w1 \\
\hline $4 \mathrm{~S}$ & $\mathrm{P} 1$ & 78001219 & 7.3 & 23 & 256 & 1.18 & $3.4 \times 10^{-16}$ & 0.60 & d & $\mathrm{D}$ & w1 \\
\hline $4 \mathrm{~S}$ & $\mathrm{P} 1$ & 78001220 & 10.0 & 52 & 64 & 1.29 & $1.8 \times 10^{-15}$ & 0.18 & c & $\mathrm{F}$ & \\
\hline $4 \mathrm{~S}$ & $\mathrm{P} 1$ & 78001221 & 11.3 & 52 & 64 & 1.79 & $3.3 \times 10^{-15}$ & 0.19 & $\mathrm{c}$ & $\mathrm{F}$ & \\
\hline $4 \mathrm{~S}$ & $\mathrm{P} 1$ & 78001222 & 12.8 & 52 & 64 & 2.15 & $1.4 \times 10^{-15}$ & 0.14 & $\mathrm{c}$ & $\mathrm{F}$ & w1 \\
\hline $4 \mathrm{~S}$ & $\mathrm{P} 1$ & 78001223 & 15.0 & 52 & 64 & 3.20 & $5.3 \times 10^{-16}$ & 0.25 & c & $\mathrm{F}$ & \\
\hline $4 \mathrm{~S}$ & $\mathrm{P} 2$ & 78001224 & 25.0 & 99 & 64 & 0.94 & $1.3 \times 10^{-14}$ & 0.23 & c & M & \\
\hline $4 \mathrm{~S}$ & $\mathrm{C} 1$ & 78001216 & 60.0 & 135 & 64 & 52.42 & $2.1 \times 10^{-15}$ & 1.13 & d & $\mathrm{F}$ & w1 \\
\hline $4 \mathrm{~S}$ & $\mathrm{C} 1$ & 78001217 & 100.0 & 135 & 64 & 84.06 & $2.2 \times 10^{-15}$ & 0.78 & c & M & \\
\hline $4 \mathrm{~S}$ & $\mathrm{C} 2$ & 78001218 & 170.0 & 180 & 64 & 24.52 & $7.0 \times 10^{-15}$ & 0.12 & c & M & \\
\hline
\end{tabular}

Table 4. Offsets of Hale-Bopp's position $\left(\mathrm{HB}_{\mathrm{pos}}\right)$ from the observed position $\left(\mathrm{HB}_{\mathrm{obs}}\right)$, and the Hale-Bopp-shadow positions offset.

\begin{tabular}{ccccc}
\hline Obs. & HB obs date & shadow date & $\left.\mathrm{HB}_{\text {pos }}-\mathrm{HB}_{\text {obs }}{ }^{\prime \prime}\right]$ & $\mathrm{HB}_{\text {obs-shadow }}\left[{ }^{\prime \prime}\right]$ \\
\hline 0 & 25-Mar.-1996 & 30-Mar.-1996 & 8.0 & 0.5 \\
1 & 27-Apr.-1996 & 5-May-1996 & 10.0 & 0.4 \\
$2 \mathrm{a}$ & 27-Sep.-1996 & 6-Sep.-1996 & 1.9 & 9031 \\
$2 \mathrm{~b}$ & 27-Sep.-1996 & 10-Oct.-1996 & 1.9 & 1811 \\
3 & 7-Oct.-1996 & 10-Oct.-1996 & 2.9 & 1.4 \\
4 & 30-Dec.-1997 & 4-Jan.-1998 & 23.8 & 27.4 \\
\hline
\end{tabular}

ejection from the nucleus at a constant rate and with constant speed yields a $1 / \rho$-dependence of the brightness of the inner coma, where $\rho$ is the projected distance from the nucleus (e.g. A'Hearn et al. 1984). This is independent of the speed: different dust populations with different, but individually constant, speeds will lead to a $1 / \rho$-distribution of the total coma brightness. Even if dust is emitted radially from only a part of the surface, a $1 / \rho$-distribution will result. (The dust acceleration region within $\sim 10$ nuclear radii is hidden within the instrumental resolution element.) Therefore, the brightness within a given aperture centered on the nucleus should scale with the aperture radius. 
Table 5. Conversion and correction factors of Hale-Bopp observations. The airy disk diameter is given for comparison with the aperture diameter. The conversion factor $C_{1}$ assumes a spectrum with $\nu \cdot F_{\nu}=$ const. The offset correction factors, $c_{\mathrm{offsetX}}$, refer to offset of observation number X. The factor $c_{\text {psf }}$ takes into account the difference of a point source and a $1 / \rho$-coma, and, finally, $c_{\mathrm{a} 23}$ converts the $1 / \rho$-coma brightness to that observed through a $23^{\prime \prime}$ diameter aperture.

\begin{tabular}{ccccccccccc}
\hline$\lambda[\mu \mathrm{m}]$ & $\begin{array}{c}\text { Aperture } \\
\text { diam. }\left[^{\prime \prime}\right]\end{array}$ & $\begin{array}{c}\text { Airy disk } \\
\text { diam. }\left[^{\prime \prime}\right]\end{array}$ & $C_{1}[\mathrm{~W} / \mathrm{Jy}]$ & $c_{\text {offset0 }}$ & $c_{\text {offset } 1}$ & $c_{\text {offset2 }}$ & $c_{\text {offset3 }}$ & $c_{\text {offset } 4}$ & $c_{\mathrm{psf}}$ & $c_{\mathrm{a} 23}$ \\
\hline 3.6 & 23 & 2.77 & $7.298 \times 10^{-15}$ & 1.151 & 1.301 & 1.006 & 1.015 & 3.884 & 1.022 & 1 \\
7.3 & 23 & 6.24 & $9.952 \times 10^{-15}$ & 1.159 & 1.313 & 1.006 & 1.015 & 3.776 & 1.051 & 1 \\
10 & 52 & 8.39 & $2.622 \times 10^{-15}$ & 1.026 & 1.042 & 1.001 & 1.002 & 1.369 & 1.026 & 0.442 \\
11.3 & 52 & 9.54 & $4.061 \times 10^{-15}$ & 1.026 & 1.042 & 1.001 & 1.002 & 1.371 & 1.030 & 0.442 \\
12.8 & 52 & 10.78 & $1.778 \times 10^{-15}$ & 1.026 & 1.042 & 1.001 & 1.002 & 1.373 & 1.034 & 0.442 \\
15 & 52 & 12.72 & $9.594 \times 10^{-16}$ & 1.027 & 1.043 & 1.001 & 1.003 & 1.377 & 1.040 & 0.442 \\
25 & 99 & 20.00 & $4.763 \times 10^{-15}$ & 1.006 & 1.01 & 1 & 1.001 & 1.066 & 1.030 & 0.232 \\
60 & 135 sqr. & 51.07 & $7.037 \times 10^{-16}$ & 1.005 & 1.007 & 1 & 1.001 & 1.038 & 1.054 & 0.162 \\
100 & 135sqr. & 86.94 & $9.443 \times 10^{-16}$ & 1.005 & 1.008 & 1 & 1.001 & 1.041 & 1.091 & 0.162 \\
170 & 180sqr. & 146.16 & $9.709 \times 10^{-16}$ & 1.001 & 1.002 & 1 & 1 & 1.014 & 1.092 & 0.120 \\
\hline
\end{tabular}

Only at distances towards the Sun of typically $10^{5} \mathrm{~km}$ from the nucleus (comparable to the projected ISOPHOT apertures) radiation pressure deflects trajectories of small particles. For big and slow particles, solar gravity may cause significant bending of dust trajectories if they stay longer than about 100 days within the region of interest. Both effects may lead to deviations from a $1 / \rho$ distribution at large distances from the nucleus. A variable dust emission rate from the nucleus also affects the brightness distribution.

In order to determine the radial brightness gradient, we have analyzed ISOCAM and ground-based images taken near the time of the ISOPHOT observations (Table 2). The ISOCAM observations were taken with the LW9 filter $\left(\lambda_{\text {eff }}=15.0 \mu \mathrm{m}\right)$ with a pixel field-of-view of $1.5^{\prime \prime}$. Azimuthally averaged radial profiles of the coma were obtained out to about $18^{\prime \prime}$ from the actual comet position. Both observations (on 1 and 7 October 1996) were fitted by a simple intensity model of the inner coma: $I_{\text {pix }} \propto \rho^{-\alpha}$, where $I_{\text {pix }}$ is the surface brightness at the radial distance, $\rho$, from the nucleus in the image plane. We found $\alpha=1.03$ for 1 Oct. and $\alpha=1.02$ for 7 Oct.

The analysis of ground-based CCD observations by Boehnhardt and Delahodde (Sect. 2) in the $R$ band over the full aperture up to $90^{\prime \prime}$ radius also displayed an approximate $1 / \rho$ profile of the surface brightness (Fig. 1). For each image, the mean flux was determined in concentric ring apertures around the coma center. The aperture series covered the diameter range of the ISOPHOT diaphragms and used a ring width of about $1^{\prime \prime}$. Stars shining through the coma were ignored using an iterative kappasigma clipping procedure (as described in ESO European Southern Observatory, 1998, Munich Image Data and Analysis System (MIDAS) Volume B: Data Reduction, copyright 1998 ESO). During the ring flux calculation the clipping procedure compares the flux in each pixel with the mean flux per pixel in the aperture ring and deletes all pixel values from the subsequent iteration which deviate by more than kappa $\times$ sigma from the mean flux per ring pixel. This process is very efficient in deleting bright stars, cosmic rays and dead or hot pixels from the flux measurements. However, it leaves very faint stars in the aperture unaffected if the summed pixel flux from the star plus the coma is not above kappa $\times$ sigma of the mean level in the aperture ring. Tests have shown that kappa values of 2-3 give the best results (with $3-4$ iterations); the effect from remaining background stars on the total ring flux is estimated to be below 1 percent. The total ring flux was computed by multiplying the mean flux per pixel by the number of pixels in the aperture ring. Finally, the ring fluxes were integrated over the diameter of the respective ISOPHOT apertures and transformed from filter mag to Jansky units at an effective wavelength of $0.65 \mu \mathrm{m}$, according to the equation,

$m[\mathrm{mag}]=-2.5 \log (F[\mathrm{Jy}] / E)$,

where $E=2870 \mathrm{Jy}$ at $\lambda_{\text {eff }}=0.65 \mu \mathrm{m}$ (Lèna et al. 1998). The resulting total fluxes, plotted in Fig. 1, are related to the radial brightness gradient, $\alpha$, as $F \propto \rho^{2-\alpha}$. From these observations, we obtain alpha-values and fluxes given in Table 6.

Since the radial profiles at both visible ( $R$ band) and infrared $(15 \mu \mathrm{m})$ wavelengths were close to $1 / \rho$ - profiles, we will assume in the following analysis that the surface brightness varied as $1 / \rho$ at all ISOPHOT wavelengths (3.6 to $170 \mu \mathrm{m})$. However, this first analysis does not take into account the observed azimuthal brightness variations which may affect offset corrections described below. Also, effects on the radial brightness profile of the neck-line antitail observed in December 1997 are not considered here.

\subsection{Offset corrections}

For the derivation of the above corrections, we have assumed that Hale-Bopp was perfectly centered in the aperture during the observations. However, the large offsets, particularly in December 1997 (Table 4), require big 


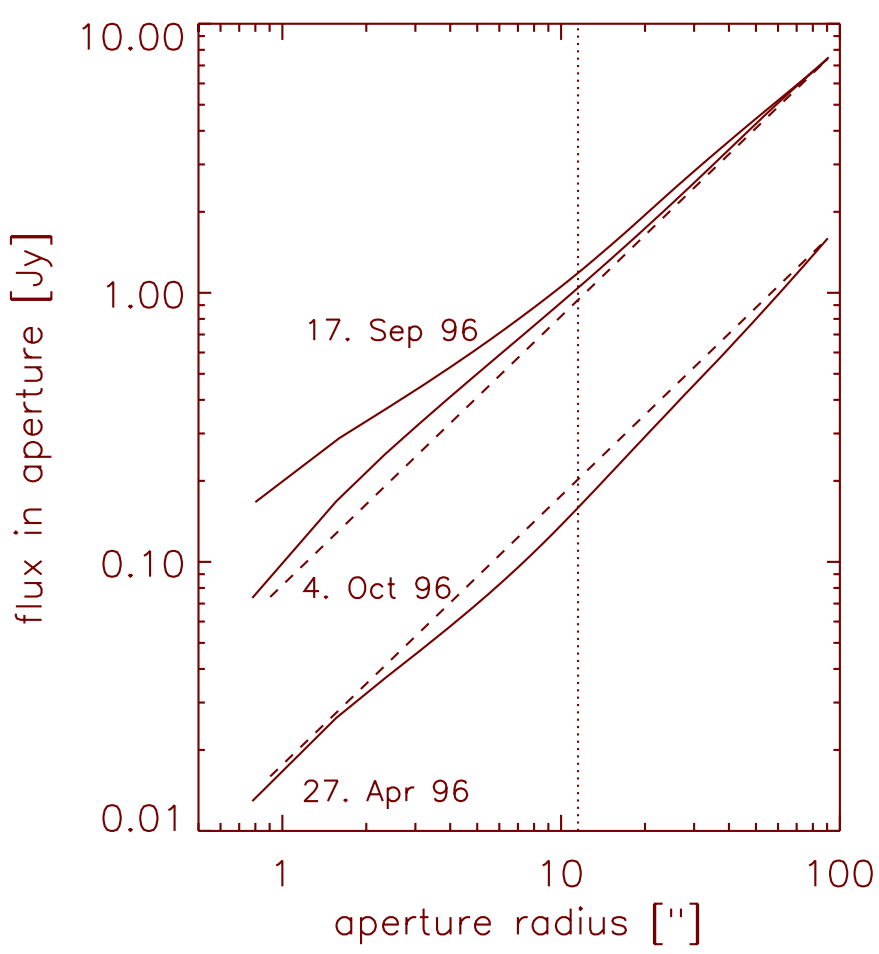

Fig. 1. Radial flux profiles of Hale-Bopp coma in the $R$ band (solid lines). Dashed line are $1 / \rho$ profiles. The dotted line marks the aperture radius for normalization.

correction factors, especially at short wavelengths, in order to calculate the flux for a centered observation. A $1 / \rho$ brightness gradient was assumed for these correction factors. For the $C_{1}$-detector, the offset has been assumed to be in the direction of $22.5^{\circ}$ relative to an array symmetry axis. This way the effect of the gaps between the pixels is about half-way between both extremes. Table 5 shows the calculated correction factors, $c_{\text {offset }}$, for all five Hale-Bopp observations.

\subsection{PSF/coma correction}

The following corrections concern effects of the pointspread-function (PSF) on coma measurements. The ISO PSF used assumes a uniformly illuminated round mirror of $30 \mathrm{~cm}$ radius with an $\mathrm{f} / 15$ focal length and a central obscuration (secondary mirror) of $10 \mathrm{~cm}$ radius. The diameter of the Airy disk of the ISO telescope is

$d_{\text {Airy }}\left[{ }^{\prime \prime}\right]=0.77 \lambda[\mu \mathrm{m}]$.

Aperture diameters of ISOPHOT used for the comet observations ranged from $23^{\prime \prime}$ to $180^{\prime \prime}$ for the different wavelengths. The ratio of the aperture size to the Airy disk varied from about 8 at the shortest wavelength to about 1.3 at the longest wavelength. Especially at the longest wavelengths, the effect of the wide PSF on the power received at the detector needs to be corrected for in order to be comparable with the measurements at shorter wavelengths.

Figure 2 compares an ideal $1 / \rho$-coma with an image of a $1 / \rho$-coma at a resolution of about $40^{\prime \prime}$ and the
Table 6. Radial slopes $\alpha$ in the $\rho$-range from $11.5^{\prime \prime}$ to $90^{\prime \prime}$ and fluxes in a $23^{\prime \prime}$ diameter aperture of the CCD observation by Boehnhardt and Delahodde (cf. Fig. 1).

\begin{tabular}{cccc}
\hline Date & 27-Apr.-96 & 17-Sep.-96 & and 24-Oct.-96 \\
\hline$\alpha$ & 0.88 & 1.11 & 1.05 \\
Flux [Jy] & 0.16 & 1.18 & 1.04 \\
\hline
\end{tabular}

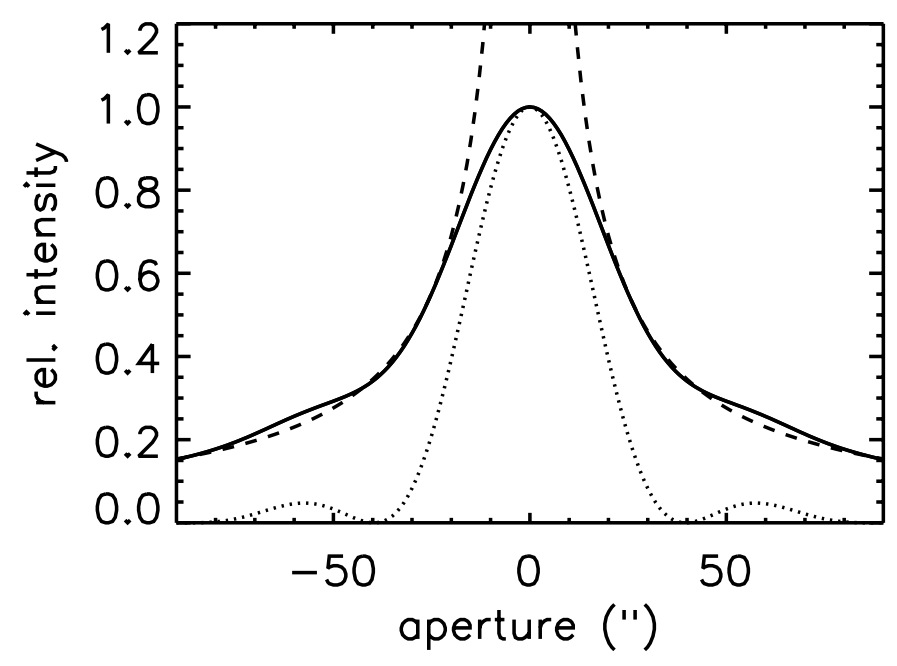

Fig. 2. PSF (dotted), $1 / \rho$-coma (dashed), PSF-broadened image of a $1 / \rho$ coma for $\lambda=100 \mu \mathrm{m}$ (solid). The singularity in the center of the $1 / \rho$ coma has been removed by the integration over a small area.

corresponding point-spread-function. While the $1 / \rho$-coma brightness is higher in the center (within about $15^{\prime \prime}$ radius), the corresponding coma image displays an excess brightness at larger distances from the center. Therefore, the integrated brightness of the coma image within a given circular aperture is significantly different from that of an ideal $1 / \rho$-coma. The PSF correction factor (cf. Table 5 ) is defined as the ratio of the brightness of an ideal $1 / \rho$ coma within the appropriate aperture size and a PSFbroadened image of a $1 / \rho$-coma. The PSF correction for C200 is smaller than expected because the central peak of the $1 / \rho$ coma is more reduced by the central gaps in between the four pixels than the flatter coma image.

\subsection{Aperture size}

The fluxes have been normalized to a standard aperture diameter of $23^{\prime \prime}$, assuming that the coma brightness scales linearly with aperture diameter (Sect. 3.4). For the rectangular apertures, we have projected the PSF-broadened image of a $1 / \rho$ coma onto the detector and calculated the flux received by all pixels. Then, we compared this flux with the flux from an ideal $1 / \rho$ coma in a $23^{\prime \prime}$ aperture. This ratio is given as $c_{\mathrm{a} 23}$ in Table 5 .

\subsection{Color correction}

ISOPHOT standard data processing leads to monochromatic fluxes (Jy) at a reference filter wavelength for an 
Table 7. Hale-Bopp color correction factors $c_{\operatorname{color} X}(X$ denotes the observation).

\begin{tabular}{cccccc}
\hline$\lambda[\mu \mathrm{m}]$ & $c_{\text {color } 0}$ & $c_{\text {color } 1}$ & $c_{\text {color } 2}$ & $c_{\text {color } 3}$ & $c_{\text {color } 4}$ \\
\hline 3.6 & 0.996 & 0.996 & 1.000 & 1.005 & 0.995 \\
7.3 & 1.163 & 1.279 & 1.054 & 1.061 & 1.050 \\
10.0 & 0.910 & 0.899 & 0.923 & 0.912 & 0.900 \\
11.3 & --- & --- & --- & --- & 1.014 \\
12.8 & 1.023 & 1.001 & 1.077 & 1.061 & 1.029 \\
15.0 & 0.957 & 1.001 & 0.898 & 0.973 & 0.979 \\
25.0 & --- & 0.920 & --- & 0.899 & 0.919 \\
60.0 & 0.904 & 0.920 & 0.962 & 1.056 & 1.026 \\
100.0 & 1.058 & 1.246 & 1.143 & 1.185 & 1.108 \\
170.0 & 1.298 & 1.130 & 1.179 & 1.157 & 1.130 \\
\hline
\end{tabular}

assumed constant energy spectrum $\nu \cdot F_{\nu}=$ const. The color correction tables for ISOPHOT contain correction factors for a black body spectrum with a given temperature, modified black body spectra (with $\nu$ or $\nu^{2}$ ) or other power law spectra. Here we produce "real" color corrections from our multi-filter measurements, by iteratively convoluting the observed spectra with the filter transmission curves. For each filter, both the observed spectral energy distribution (first, without color correction applied) and the $\nu \cdot F_{\nu}$ power law spectrum are normalized at the ISOPHOT filter central wavelength. Both normalized spectra are then integrated over the filter band pass and the ratio of both integrals is the color correction factor for the corresponding observation and wavelength. In a second and any further iteration the color-corrected spectral energy distribution is used. After a few iterations the color correction factors (Table 7) are stable (deviations between subsequent iterations are $<10^{-3}$ ). The derived color correction factors are all reasonably close to 1.0 , with $0.8<c_{\text {color }}<1.3$.

\subsection{Flux calculation}

The true flux, $F_{\nu}$, is calculated from the observed IBP by applying all corrections described above:

$$
\begin{array}{r}
F_{\nu}=\left(\mathrm{IBP}_{\mathrm{HB}}-\mathrm{IBP}_{\text {shadow }}\right) \times c_{\mathrm{offset} X} \times c_{\mathrm{PSF}} \\
\times c_{\mathrm{color} X} \times c_{\mathrm{a} 23} / C_{1}
\end{array}
$$

where $C_{1}$ is a constant for each detector. The final, calibrated fluxes for Hale-Bopp are presented in Table 8.

\section{Discussion}

\subsection{Spectral energy distribution}

The calibrated fluxes for the five data sets are plotted in Fig. 3. The thermal spectral energy distribution (SED) over the broad spectral range covered by the ISOPHOT data provides information about the size and composition of the particles in the coma at the time of observation. Since small particles do not radiate efficiently at wavelengths more than about ten times their radius, comparison of the flux at $100-170 \mu \mathrm{m}$ with that at shorter wavelengths gives an estimate of the relative cross-section
Table 8. Spectral energy distributions of Hale-Bopp observations. Fluxes and $1-\sigma$ minimum and maximum fluxes are given for each filter. The fluxes correspond to a $23^{\prime \prime}$ effective

\begin{tabular}{|c|c|c|c|c|}
\hline Obs. date & $\begin{array}{c}\lambda \\
{[\mu \mathrm{m}]}\end{array}$ & $\begin{array}{c}\text { Flux } \\
{[\mathrm{Jy}]}\end{array}$ & $\begin{array}{l}\text { Min. } \\
\text { Flux } \\
{[\mathrm{Jy}]}\end{array}$ & $\begin{array}{c}\text { Max. } \\
\text { Flux } \\
{[\mathrm{Jy}]}\end{array}$ \\
\hline \multirow{8}{*}{ 25-Mar.-96 } & 3.6 & 0.025 & 0.0 & 0.025 \\
\hline & 7.3 & 0.603 & 0.4 & 0.91 \\
\hline & 10.0 & 3.49 & 2.76 & 4.40 \\
\hline & 12.8 & 4.87 & 3.62 & 6.55 \\
\hline & 15.0 & 7.15 & 5.82 & 8.78 \\
\hline & 60.0 & 10.9 & 6.65 & 18.0 \\
\hline & 100 & 4.38 & 2.89 & 6.64 \\
\hline & 170 & 1.06 & 0.883 & 1.29 \\
\hline \multirow[t]{9}{*}{ 27-Apr.-96 } & 3.6 & 0.025 & 0.0 & 0.025 \\
\hline & 7.3 & 0.579 & 0.343 & 0.975 \\
\hline & 10.0 & 4.63 & 3.85 & 5.57 \\
\hline & 12.8 & 7.04 & 5.34 & 9.26 \\
\hline & 15.0 & 9.37 & 7.80 & 11.2 \\
\hline & 25.0 & 21.2 & 17.8 & 25.3 \\
\hline & 60.0 & 11.8 & 8.42 & 16.7 \\
\hline & 100 & 2.55 & 1.76 & 3.70 \\
\hline & 170 & 1.26 & 1.05 & 1.52 \\
\hline \multirow[t]{8}{*}{ 27-Sep.-96 } & 3.6 & 0.315 & 0.105 & 0.944 \\
\hline & 7.3 & 9.58 & 6.28 & 14.6 \\
\hline & 10.0 & 39.9 & 27.8 & 57.4 \\
\hline & 12.8 & 51.4 & 37.0 & 71.5 \\
\hline & 15.0 & 102 & 79.6 & 132 \\
\hline & 60.0 & 29.6 & 18.1 & 48.5 \\
\hline & 100 & 9.14 & 4.75 & 17.5 \\
\hline & 170 & 3.54 & 2.86 & 4.37 \\
\hline \multirow[t]{9}{*}{ 07-Oct.-96 } & 3.6 & 0.256 & 0.085 & 0.768 \\
\hline & 7.3 & 10.0 & 7.31 & 13.9 \\
\hline & 10.0 & 45.0 & 38.3 & 52.9 \\
\hline & 12.8 & 53.2 & 43.6 & 64.9 \\
\hline & 15.0 & 90.8 & 72.2 & 114 \\
\hline & 25.0 & 203 & 162 & 255 \\
\hline & 60.0 & 30.4 & 19.6 & 47.2 \\
\hline & 100 & 8.08 & 4.30 & 15.1 \\
\hline & 170 & 3.48 & 2.84 & 4.26 \\
\hline \multirow[t]{10}{*}{ 30-Dec.-97 } & 3.6 & 0.153 & 0.001 & 0.153 \\
\hline & 7.3 & 3.40 & 2.09 & 5.53 \\
\hline & 10.0 & 13.2 & 10.2 & 17.1 \\
\hline & 11.3 & 12.6 & 8.83 & 17.9 \\
\hline & 12.8 & 15.1 & 12.6 & 18.1 \\
\hline & 15.0 & 23.6 & 18.2 & 30.5 \\
\hline & 25.0 & 49.4 & 37.1 & 65.8 \\
\hline & 60.0 & 13.5 & 6.30 & 28.9 \\
\hline & 100 & 5.36 & 2.89 & 9.94 \\
\hline & 170 & 2.66 & 2.32 & 3.05 \\
\hline
\end{tabular}
aperture.

in large and small particles. Solid state spectral features, particularly for silicates, also occur within the ISOPHOT spectral range.

A useful first step in assessing the data is to compare the spectral energy distribution with that of a blackbody. Departures from blackbody emission can then be interpreted in terms of particle emission properties. 

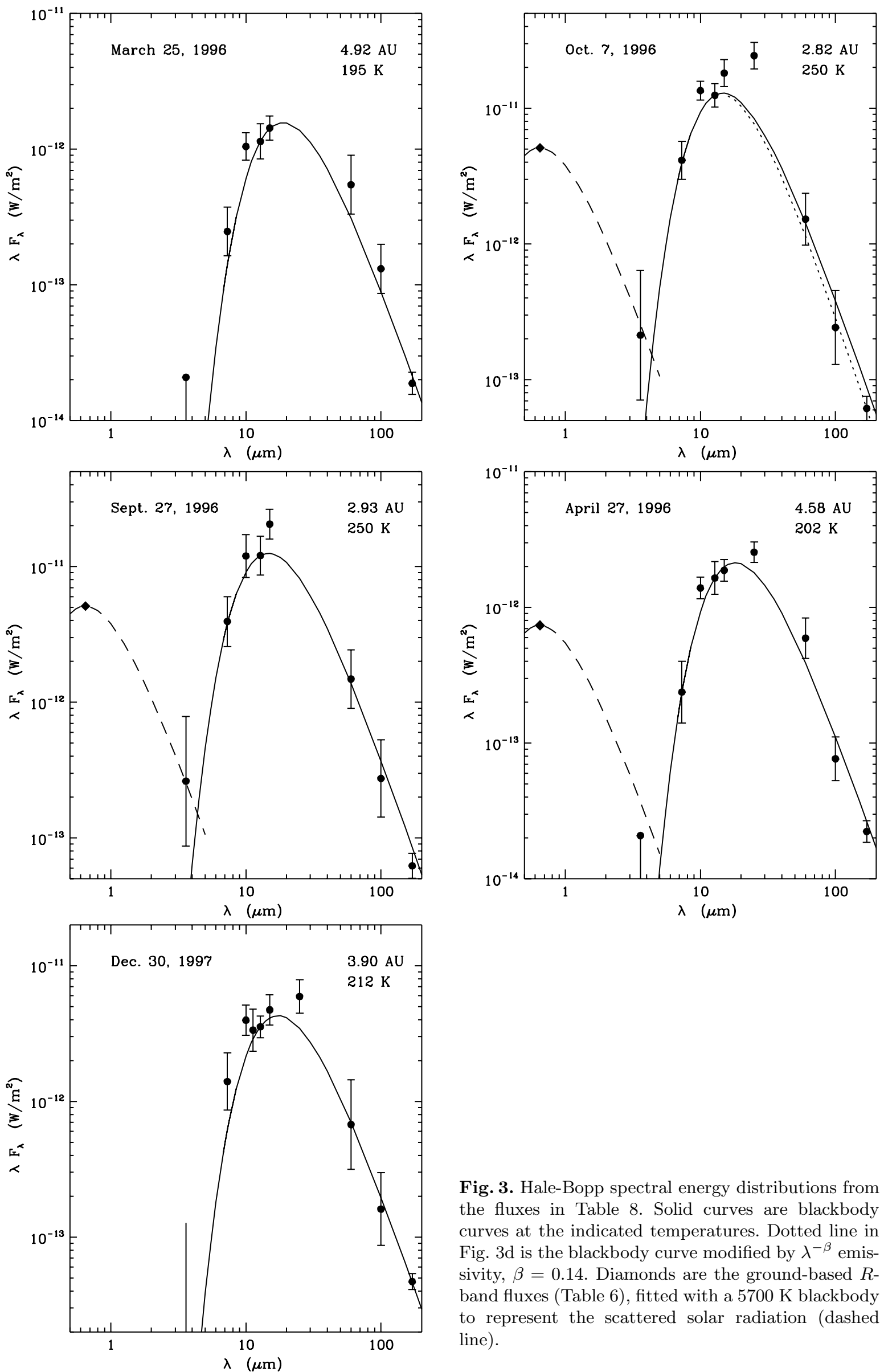

Fig. 3. Hale-Bopp spectral energy distributions from the fluxes in Table 8. Solid curves are blackbody curves at the indicated temperatures. Dotted line in Fig. $3 \mathrm{~d}$ is the blackbody curve modified by $\lambda^{-\beta}$ emissivity, $\beta=0.14$. Diamonds are the ground-based $R$ band fluxes (Table 6), fitted with a $5700 \mathrm{~K}$ blackbody to represent the scattered solar radiation (dashed line). 
This comparison also allows us to gain confidence in the photometry, by ascertaining whether flux values make sense physically. Reference blackbody curves are compared with the photometry in Fig. 3; the following discussion is based on these figures.

First, all five data sets display similar patterns in the shape of the SED. Note particularly the good correspondence between 27 September and 7 October 1996. The data for March and April 1996 also agree well, except at $100 \mu \mathrm{m}$. The consistent shape of the SED means that random errors in the data are not large and that the average size and composition of the dust in the coma did not change drastically over time.

Next, it is evident that the data points in any data set do not all follow a smooth Planck function of any temperature. In particular, there is excess emission at 10 and $25 \mu \mathrm{m}$, consistent with the silicate emission features seen in ISO and ground-based spectroscopic data. The silicate emission is present even in March and April 1996 at 4.64.9 AU, the largest heliocentric distance at which silicate emission has ever been observed in a comet.

In order to compare the SED with a blackbody, one has to decide which bandpasses sample the continuum. Here, we are aided by the ISO SWS and LWS spectra taken near the time of our Sept.-Oct. data (Fig. 4) and a PHT-S spectrum taken in April 1996. The $3.6 \mu \mathrm{m}$ flux contains both thermal emission and scattered light. No emission feature is seen near $7.3 \mu \mathrm{m}$ in the spectra, as this bandpass samples the thermal emission dust continuum. The flux at $12.8 \mu \mathrm{m}$ will be close to the continuum, with a small contribution from the long-wavelength side of the $10 \mu \mathrm{m}$ silicate feature. In ground-based spectra of HaleBopp, the flux is down to the continuum level at $\sim 12.5 \mu \mathrm{m}$ (Hayward et al. 2000). The $15 \mu \mathrm{m}$ filter is near the onset of the strong $20 \mu \mathrm{m}$ silicate emission feature in the SWS spectrum. Water ice has a broad feature near $65 \mu \mathrm{m}$ that appears to be present in the LWS spectrum (Lellouch et al. 1998). Both 100 and $170 \mu \mathrm{m}$ should be free of spectral features. For the purpose of this discussion, we have generally fitted a reference blackbody through the 7.3 and $12.8 \mu \mathrm{m}$ points. However, the $7.3 \mu \mathrm{m}$ measurements were taken through a smaller aperture than the other data, so we are relying on the accuracy of the aperture correction factors.

The temperature of a blackbody fit to the data over a limited spectral range will be referred to as a color temperature, $T_{\mathrm{c}}$, and will be compared to the temperature of a rotating blackbody in equilibrium with the solar radiation, $T_{\mathrm{bb}}=278 r^{-0.5}$.

\section{March and 27 April 1996}

At 4.9-4.6 AU, these are the most distant thermal infrared observations of comet Hale-Bopp. Fitting a Planck function through the 7.3 and $12.8 \mu \mathrm{m}$ points on 27 April yields $T_{\mathrm{c}}=202 \mathrm{~K}$ and $T_{\mathrm{c}} / T_{\mathrm{bb}}=1.55$. Although the $7.3 \mu \mathrm{m}$ error bar is large, this fit does fall within $1-\sigma$ at both 100 and $170 \mu \mathrm{m}$. The fluxes at 10 and $25 \mu \mathrm{m}$ (silicate bands) are a factor $\sim 1.5$ above the continuum. A lower color temperature would cause the $25 \mu \mathrm{m}$ point to lie close to the continuum, while increasing the silicate excess at $10 \mu \mathrm{m}$. The flux at $60 \mu \mathrm{m}$ is above the continuum, suggesting the presence of water ice emission (Sect. 4.4).

A PHT-S 6-11.7 $\mu \mathrm{m}$ spectrum was taken on 27 April (Crovisier et al. 1996). It shows a silicate feature about a factor 2 above the continuum fit at $6-8 \mu \mathrm{m}$. The $T_{\mathrm{c}}$ derived from the $6-8 \mu \mathrm{m}$ continuum is $\sim 162 \mathrm{~K}$, much lower than the fit to our photometry. Such a low color temperature is not compatible with our data set on 27 April. While the $10 \mu \mathrm{m}$ excess would be large, the corresponding $25 \mu \mathrm{m}$ flux would actually fall below the continuum. Moreover, the 100 and $170 \mu \mathrm{m}$ points would be a factor $\sim 3.5$ below the extrapolated continuum.

A literal fit of a Planck function through the 7.3 and $12.8 \mu \mathrm{m}$ points on 25 March would give $T_{\mathrm{c}}=225 \mathrm{~K}$ and $T_{\mathrm{c}} / T_{\mathrm{bb}}=1.79$, higher than any other data set. The 100 and $170 \mu \mathrm{m}$ data points would lie high above the extrapolated blackbody continuum, inconsistent with other data sets. Instead, we make the plausible assumption that the coma is similar to 27 April and adopt $T_{\mathrm{c}} / T_{\mathrm{bb}}=1.55$, or $T_{\mathrm{c}}=195 \mathrm{~K}$. This curve fits the data at $12.8,15$, and $170 \mu \mathrm{m}$. The $10 \mu \mathrm{m}$ flux/continuum ratio is $\sim 1.7$, similar to that on 27 April. Because of the uncertainty in placing the continuum, and because there is no $25 \mu \mathrm{m}$ data point in March, the April 1996 data set is more reliable for interpreting grain properties at large heliocentric distance.

\section{September and 7 October 1996}

By the time the comet reached 2.9-2.8 AU, the dust grains were warmer and the thermal emission considerably stronger. The photometry on the two dates is very consistent, well within the uncertainties. The blackbody curve fitting the 7.3 and $12.8 \mu \mathrm{m}$ fluxes corresponds to $T_{\mathrm{c}}=250 \mathrm{~K}$, on both dates. The ratio $T_{\mathrm{c}} / T_{\mathrm{bb}}=1.5$. For this $T_{\mathrm{c}}$, the $10 \mu \mathrm{m}$ silicate feature is a factor 1.5 above the continuum, while the $25 \mu \mathrm{m}$ flux is a factor 3 above the continuum. Comparison of the photometry with the ISO spectra (Fig. 4) suggests that the ISOPHOT $10 \mu \mathrm{m}$ flux may be $\sim 1-\sigma$ too low. Ground-based 8-13 $\mu$ m spectra on 29-30 September have a $10 \mu \mathrm{m}$ total flux/continuum ratio of $\sim 2$ and an $8-13 \mu \mathrm{m} T_{\mathrm{c}} \sim 240 \mathrm{~K}$ (Hayward et al. 2000). For October 31, 1996, Lisse et al. (1999) find a ratio $T_{\mathrm{c}} / T_{\mathrm{bb}} \sim 2$, with the $10 \mu \mathrm{m}$ flux about 2 to 3 times the nearby continuum flux.

\section{December 1997}

The color temperature required to fit the 7.3 and $12.8 \mu \mathrm{m}$ data points with a Planck function is extremely high $(260 \mathrm{~K})$ and leaves the $60-170 \mu \mathrm{m}$ data points well above the extrapolated "continuum". However, this observation was mis-pointed by $24^{\prime \prime}$. The offset correction factor of 3.8 at $7.3 \mu \mathrm{m}$ is far larger than the corrections for the longer wavelengths having larger apertures. The $11.3-25 \mu \mathrm{m}$ photometry is consistent with the SWS spectrum acquired on 28 December, while the corrected $7.3 \mu \mathrm{m}$ flux is a factor of $3-4$ higher than the spectrum. A prominent neck-line structure (formed by large grains 
released around perihelion) extended right through the inner coma in visual-wavelength images taken at this time (Boehnhardt private communication) and may have distorted the $1 / \rho$ brightness distribution. A Planck function for $T_{\mathrm{c}}=215 \mathrm{~K}$ gives $T_{\mathrm{c}} / T_{\mathrm{bb}} \sim 1.5$, similar to the other data sets, and places the $12.8,60,100$, and $170 \mu \mathrm{m}$ points on the continuum. The silicate features at 10 and $25 \mu \mathrm{m}$ are then about a factor of two above the continuum. Regardless of the uncertainty, this data set shows that the comet still had a large dust coma of small grains producing strong 10 and $25 \mu \mathrm{m}$ silicate emission and high thermal emission relative to a blackbody, even at almost 4 AU postperihelion. The error bars on the $60 \mu \mathrm{m}$ flux are too large for us to say whether icy grains were present in the coma in December 1997.

In summary, the fluxes derived from the full data reduction are, in general, physically reasonable within the error bars. The March and April 1996 photometry displays surprisingly strong thermal emission for such a large heliocentric distance. The strong thermal emission, high color temperature, and strong silicate feature contrast with our pre-Hale-Bopp expectation that at large heliocentric distances the small grains would be bound in larger icy particles.

The ratio of the $7.3-12.8 \mu \mathrm{m}$ color temperature to the equilibrium blackbody temperature, $T_{\mathrm{bb}}$, was consistently higher for Hale-Bopp than for other comets observed in the infrared, in accord with ground-based photometry (Williams et al. 1997; Mason et al. 2000). The color temperature determined by fitting a Planck function over a given wavelength interval is not equal to the physical temperature of the grains. The thermal radiation from a single grain depends on both its temperature and wavelengthdependent emissivity. The dust coma contains grains of differing size, composition, and temperature and what we observe is the integral of the thermal emission from all these grains. An elevated color temperature does mean that small grains with wavelength-dependent emissivity and physical temperatures considerably warmer than an equilibrium blackbody are contributing to the emission at the shorter wavelengths.

If the dust coma consisted only of small grains $(\mu \mathrm{m}-$ sub- $\mu \mathrm{m}$ radius), the flux at long wavelengths would decrease steeply compared to a blackbody, because small grains cannot radiate efficiently at wavelengths much larger than their own dimension. In the Rayleigh limit, their emissivity would decrease $\propto \lambda^{-2}$. If we fit our data by $B_{\lambda}(T) \cdot \lambda^{-\beta}$ from $12.8-170 \mu \mathrm{m}$, we find $\beta \sim 0.1$ (dashed curve in Fig. $3 \mathrm{~d}$ ). Thus, a broad range of grain sizes must have been present in the coma at 2.8-4.9 AU. A value of $\beta \sim 0.1$ agrees well with that found for other dusty comets (C/Levy 1990; Lisse et al. 1998). Jewitt \& Matthews (1999) detected a high abundance of large (submm) grains in the coma near perihelion from submillimeter observations; they determined $\beta \sim 0.6$ in the submm wavelength range.

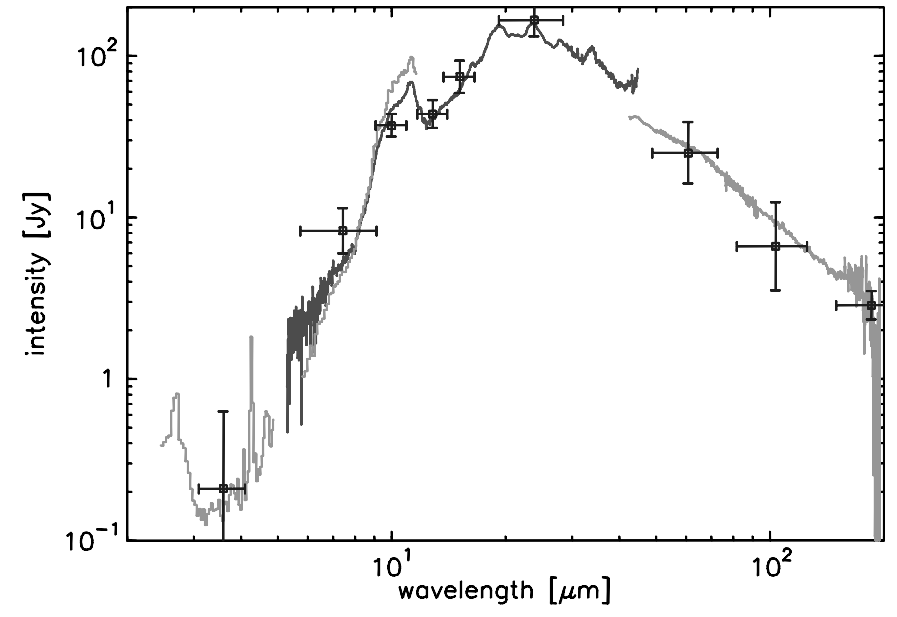

Fig. 4. Comparison of ISOPHOT fluxes (open squares) with the PHT-S, SWS, and LWS spectra on 7 October 1996. All fluxes have been scaled to a $19^{\prime \prime}$ diameter aperture.

\subsection{Comparison with ISO spectroscopy}

IR spectra of Hale-Bopp were acquired with ISO within 12 days of the photometry. Figure 4 compares the 7 October ISOPHOT fluxes with the spectra taken on 6-7 October 1996. The spectra are from PHT-S $(2.5-11.6 \mu \mathrm{m}$; Crovisier et al. 1997b), SWS (5.3-45 $\mu \mathrm{m}$; Crovisier et al. 2000) and LWS (43-195 $\mu \mathrm{m}$; Lellouch et al. 1998). The full SWS spectrum consisted of several segments observed independently with rectangular fields of view ranging from $14^{\prime \prime} \times 20^{\prime \prime}$ to $20^{\prime \prime} \times 33^{\prime \prime}$. PHT-S utilized a $24^{\prime \prime}$ square aperture and LWS a $100^{\prime \prime}$ circular aperture. All fluxes have been scaled to a $19^{\prime \prime}$ diameter aperture (corresponding to the smallest aperture of the SWS) according to a $1 / \rho$ law (thus the fluxes from Table 8 have been divided by 1.22). For SWS, additional scaling factors have been applied to the different bands of the instrument to make the spectrum continuous; these scaling factors are close to one. The horizontal bars on the photometry are the filter bandwidths. The good agreement between the independently calibrated photometry and spectroscopy is encouraging and demonstrates that the error bars assigned to the photometry are reasonable. The abrupt break between the SWS and LWS spectra near $44 \mu \mathrm{m}$ is not understood; however, the overall slope in the spectrum at longer wavelengths is consistent with the photometry and gives us confidence in our aperture correction factors.

A comparison between the 30 Dec. 1997 fluxes and PHT-S and SWS spectra taken on 25 and 28 Dec. 1997 (Crovisier et al. 1999) indicates that the offset correction factor used at 7.3 and $3.6 \mu \mathrm{m}$ is probably not correct. These two data points lie a factor of $\sim 3$ above the spectra when the correction factor has been applied. The correction factor of 1.37 for the $10-25 \mu \mathrm{m}$ data points is of the same order as the uncertainty in the comparison of the spectra and the photometry. 


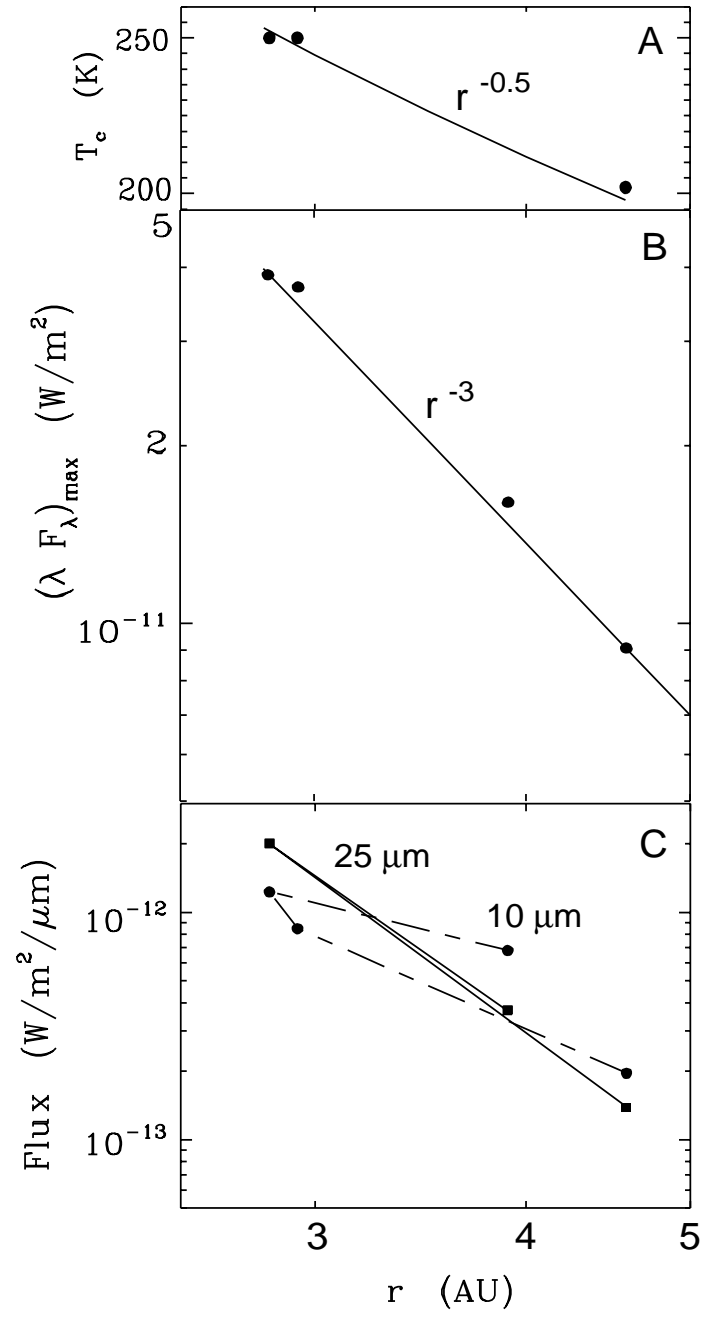

Fig. 5. Heliocentric distance variations. A. 7.3-12.8 $\mu \mathrm{m}$ color temperature. B. $\left(\lambda F_{\lambda}\right)_{\max }$, scaled to $\Delta=1 \mathrm{AU}$. This is a measure of the total thermal energy. C. $10 \mu \mathrm{m}$ and $25 \mu \mathrm{m}$ flux excess above the continuum, scaled to $\Delta=1 \mathrm{AU}$.

\subsection{Heliocentric distance dependences}

The dependence of the color temperature on heliocentric distance is plotted in Fig. 5 (top panel). The variation with $r$ is close to $r^{-0.5}$, the dependence expected for a blackbody, although the $T_{\mathrm{c}}$ are $\sim 50 \%$ higher than a theoretical blackbody. The color temperature also varied $\propto r^{-0.5}$ in comet P/Halley between 2.8 and $0.8 \mathrm{AU}$; the $8-20 \mu \mathrm{m} T_{\mathrm{c}}$ was $\sim 15 \%$ above the theoretical blackbody temperature (Tokunaga et al. 1988).

When the SED can be fitted by a Planck function, the quantity $\left(\lambda F_{\lambda}\right)_{\max }$ is proportional to the total energy radiated (Ney 1982; Gehrz \& Ney 1992). Figure 5 (middle panel) shows the heliocentric variation of $\left(\lambda F_{\lambda}\right)_{\max }$, normalized to $1 \mathrm{AU}$ geocentric distance. We find $\left(\lambda F_{\lambda}\right)_{\max } \propto$ $r^{-3}$. For a constant cross-section of radiating grains in the coma, the total energy should scale $\propto T^{4}$, thus $\propto r^{-2}$. If the dust production rate varies directly with input solar energy, then the dust cross-section in the coma should also scale $\propto r^{-2}$ and $\left(\lambda F_{\lambda}\right)_{\max } \propto r^{-4}$. The total energy typically varied $\propto r^{-4}$ in 7 comets observed by Ney (1982), as well as in P/Halley (Gehrz \& Ney 1992). The dust production rate in comet Hale-Bopp varied less steeply with $r$ between 4.6 and 2.8 AU, reflecting the high activity generated by $\mathrm{CO}$ at large $r$. The data point for 30 December 1997 falls close to the relation defined by the preperihelion values, indicating that the dust production rate postperihelion remained high as the comet receded from the Sun.

Figure 5 (bottom panel) plots the excess flux above the interpolated continuum for the spectral features at 10 and $25 \mu \mathrm{m}$. The fluxes are normalized to $1 \mathrm{AU}$ geocentric distance, but no normalization for the differing grain temperatures has been performed. The excess flux at $25 \mu \mathrm{m}$ varies as $r^{-5.5}$ from 4.6-2.8 AU and the post-perihelion value at $3.90 \mathrm{AU}$ fits with the relation defined by the preperihelion observations. The $10 \mu \mathrm{m}$ excess flux varies as $r^{-3.8}$. However, the $10 \mu \mathrm{m}$ fluxes on 27 September and 7 October appear to be $\sim 1-\sigma$ low compared to the spectra; if the $10 \mu \mathrm{m}$ flux were $1-\sigma$ higher on October 7 , the excess flux would vary $\propto r^{-5}$, closer to the $25 \mu$ m variation.

\subsection{Dust albedo}

It is possible to use the $R$-band scattered light brightness together with the thermal emission to derive an average albedo of the dust grains at 4.6 and 2.8 AU. The single scattering albedo of a particle is defined as the ratio of the energy scattered in all directions to the total energy removed from the beam (van de Hulst 1957; Hanner et al. 1981). The total energy removed from the beam corresponds to the total energy reradiated in the infrared.

One cannot determine the single scattering albedo from comet observations at a single scattering angle. However, the albedo $A(\theta)$ at the scattering angle of observation is useful for comparing the dust properties among comets. We have

$\frac{f_{\mathrm{vis}}(\theta)}{f_{\mathrm{ir}}(\theta)}=\frac{A(\theta)}{1-A(\theta)}$

where $f_{\text {vis }}$ and $f_{\text {ir }}$ are respectively the total energy scattered and thermally emitted.

Gehrz \& Ney (1992) have shown that

$\frac{f_{\mathrm{vis}}(\theta)}{f_{\mathrm{ir}}(\theta)}=\frac{\left[\lambda F_{\lambda}\right]_{\max , \mathrm{vis}}}{\left[\lambda F_{\lambda}\right]_{\max , \mathrm{ir}}}$

where $\left[\lambda F_{\lambda}\right]_{\max }$ is the Planckian maximum in the spectral energy distribution (Fig. 3). We take the $R$-band fluxes in a $23^{\prime \prime}$ aperture from Table 6 , averaging the 17 September and 24 October fluxes for 7 October, cf. $A(\theta) \sim 0.39$ on 31 October 1996, as reported by Lisse et al. (1999). Then $\frac{f_{\text {vis }}(\theta)}{f_{\text {ir }}(\theta)}$ is 0.345 on 27 April and 0.40 on 7 October, yielding $A(\theta)=0.26$ on 27 April $\left(\theta=168^{\circ}\right)$ and $A(\theta)=0.28$ on 7 October $\left(\theta=161^{\circ}\right)$. Note that this definition of albedo differs from the geometric albedo used, for example, in Tokunaga et al. (1986), defined as the ratio of the energy scattered by a particle at $180^{\circ}$ to that scattered by a white Lambertian disk of the same cross section (Hanner et al. 1981). 
These values of $A(\theta)$ can be compared with the range $0.25-0.40$ for Hale-Bopp at $\theta=140^{\circ}-160^{\circ}$ measured by Mason et al. (2000) near perihelion. The albedo of HaleBopp is higher than the typical values of $A(\theta) \sim 0.20$ found by Gehrz \& Ney (1992) for comet Halley. The albedo of a particle depends on grain size and optical properties; porosity also affects the albedo. A higher average albedo for the grains in the coma of Hale-Bopp implies smaller mean grain size and/or less absorbing grains, such as small exposed silicate grains. A correlation of higher albedo with stronger silicate feature was noted in comet Halley (Gehrz \& Ney 1992; Tokunaga et al. 1986) and the unusually strong silicate feature in Hale-Bopp supports this correlation.

For the typical phase function of cometary dust (Millis et al. 1982), the albedo should have been $\sim 15 \%$ higher on 27 April at $\theta=168^{\circ}$ than on 7 October at $\theta=161^{\circ}$. Since the albedo was, in fact, slightly lower on 27 April, we can rule out a substantial component of cold, bright icy grains enhancing the scattered light in the coma at 4.6 AU.

\subsection{Dust models}

The observed thermal emission arises from dust particles of varied sizes and composition, radiating according to their temperatures and wavelength- dependent emissivities. The temperature of each grain is determined by the balance between the solar energy absorbed at visual and ultraviolet wavelengths and the energy emitted in the infrared and will depend upon grain size and composition (e.g. Hanner 1983). In this section, we present examples of dust models compared with the ISO photometry and spectra.

The thermal spectral energy distribution has been calculated for models consisting of small (submicron) silicate grains and a broad size distribution of featureless absorbing particles. Emissivities and temperatures of the absorbing particles were computed using Mie theory and the glassy carbon optical constants of Edoh (1983). A size distribution for the absorbing grains of the form described by Hanner (1983) has been employed,

$n(a)=\left[1-a_{\mathrm{o}} / a\right]^{M}\left(a_{\mathrm{o}} / a\right)^{N}$

where $a_{\mathrm{o}}$ is the minimum grain size contributing measurably to the thermal emission, $N$ defines the slope of the size distribution at large $a$, and $M$ determines the peak grain radius, once $a_{\mathrm{o}}$ and $N$ have been specified.

After some experimentation, a mixture of $75 \%$ glassy pyroxene, $10 \%$ glassy olivine, and $15 \%$ crystalline olivine was adopted for the silicate component, to provide an approximate fit to the SWS spectral shape. The emissivities of the glassy components were computed by Mie theory from the refractive indices in Dorschner et al. (1995), while the emissivity of the crystalline olivine (forsterite) was taken directly from Koike et al. (1993). The temperature of pure silicate grains will be much colder than a blackbody. However, the grains are most likely associated with absorbing material (Kissel et al. 1986;
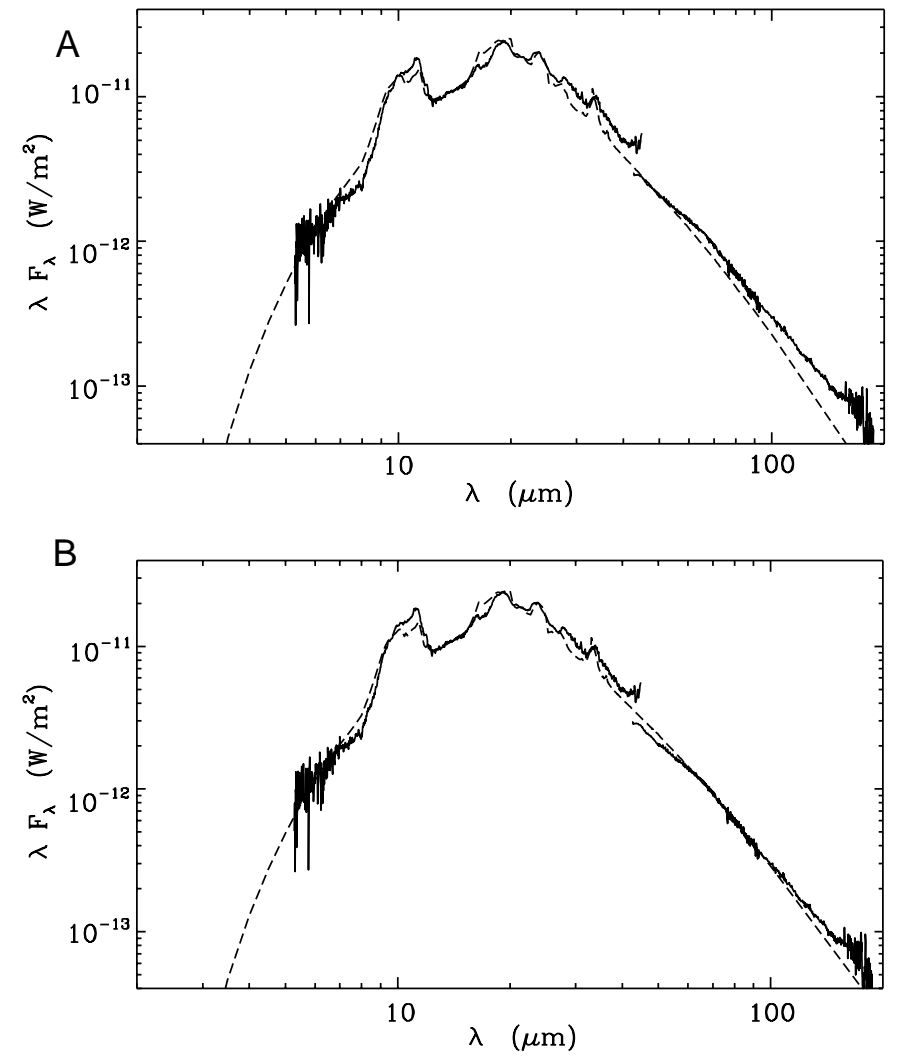

Fig. 6. Comparison of dust model (dashed curve) with the SWS and LWS spectra on 7 October 1996. A. size distribution power law, $N=3.7$. B. size distribution power law $N=3.5$. The flatter size distribution fits better at longer wavelengths.

Li \& Greenberg 1998) sufficient to make them warm. We have left the temperature of the silicate grains as a free parameter, to be determined by the observed flux ratio between the 10 and $25 \mu \mathrm{m}$ silicate features. The relative abundance of the silicate and absorbing grains is also a free parameter.

We focus on 7 October, where the existence of the full infrared spectrum provides better constraints on the model. Figure 6 (top panel) compares the ISO SWS and LWS spectra with a model using the above size distribution, with $N=3.7, a_{\mathrm{o}}=0.1 \mu \mathrm{m}$, peak grain radius $0.42 \mu \mathrm{m}$, and upper cutoff radius $1 \mathrm{~cm}$. The overall shape of the spectrum is well reproduced by the model. The relative flux at 10 and $20 \mu \mathrm{m}$ sets a temperature of $\sim 180 \mathrm{~K}$ for the silicate grains. This temperature is slightly above the theoretical blackbody temperature at $2.82 \mathrm{AU}$, indicating that the silicates are indeed associated with some absorbing material. The silicate mixture matches the spectral structure of the silicate features within $10 \%$. If the silicate grains have a typical radius of $0.5 \mu \mathrm{m}$ and $T=180 \mathrm{~K}$, then the total cross section of silicate grains is approximately equal to the total cross section of absorbing grains in this model. (The total required cross section of silicate grains scales inversely with the grain radius.)

The slope of the model at long wavelengths is steeper than the LWS spectrum, an indication that the abundance of larger grains $(\sim 100 \mu \mathrm{m})$ in the coma is higher than 


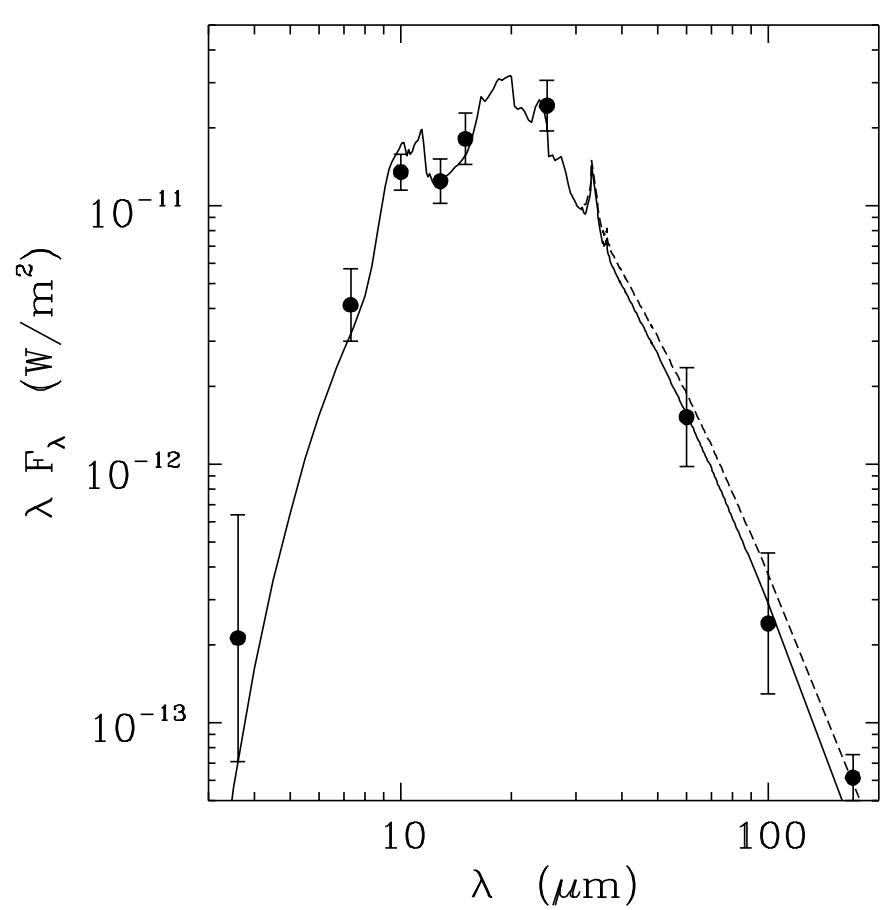

Fig. 7. Comparison of dust models with ISOPHOT data on 7 October 1996 at 2.8 AU. Solid curve: $N=3.7$, dashed curve: $N=3.5$. The models are identical to those fitted to the spectra in Fig. 6.

in the model size distribution. A flatter size distribution, $N=3.5$, is compared with the spectra in Fig. 6 (bottom panel). This model matches the overall slope of the spectra from 20 to $150 \mu \mathrm{m}$. A value of $N<4$ means that the dust mass in the coma is concentrated towards the largest particles. If the dust outflow velocity depends on grain radius as $v \propto a^{-0.5}$ (cf. Lien et al. 1992 Tempel 2 dust trail paper), then the power-law index for the dust production size distribution is $N+0.5$. Thus, $N=3.5$ is the limiting value between dust production size distributions mass weighted towards large and small particles. The mass of dust is concentrated in the largest particles in all comets measured to date (Lisse et al. 1998). The surface area can be in either the small (for optically bright, dusty comets) or large (for optically faint, "gassy" comets) grains.

Figure 7 compares the 7 October photometry with these two models, each normalized to the data at $12.8 \mu \mathrm{m}$. The model with $N=3.7$ fits within 1- $\sigma$ at all wavelengths except $170 \mu \mathrm{m}$, while the model with $N=3.5$ matches the $170 \mu \mathrm{m}$ measured flux. The slope at $7.3-10 \mu \mathrm{m}$ is a compromise between the spectrum and the photometry; the ISOPHOT $10 \mu \mathrm{m}$ flux is $1-\sigma$ below the spectrum and the $7.3 \mu \mathrm{m}$ flux $1-\sigma$ above the spectrum (Fig. 4). The $7.3 \mu \mathrm{m}$ flux in the model is sensitive to the abundance of small, hot absorbing grains and can be adjusted by shifting the peak size in the size distribution.

Figure 8 (dashed curve) shows the 27 April ISOPHOT data and the predicted fluxes at 4.6 AU for a model with the same size distribution and silicate mixture as

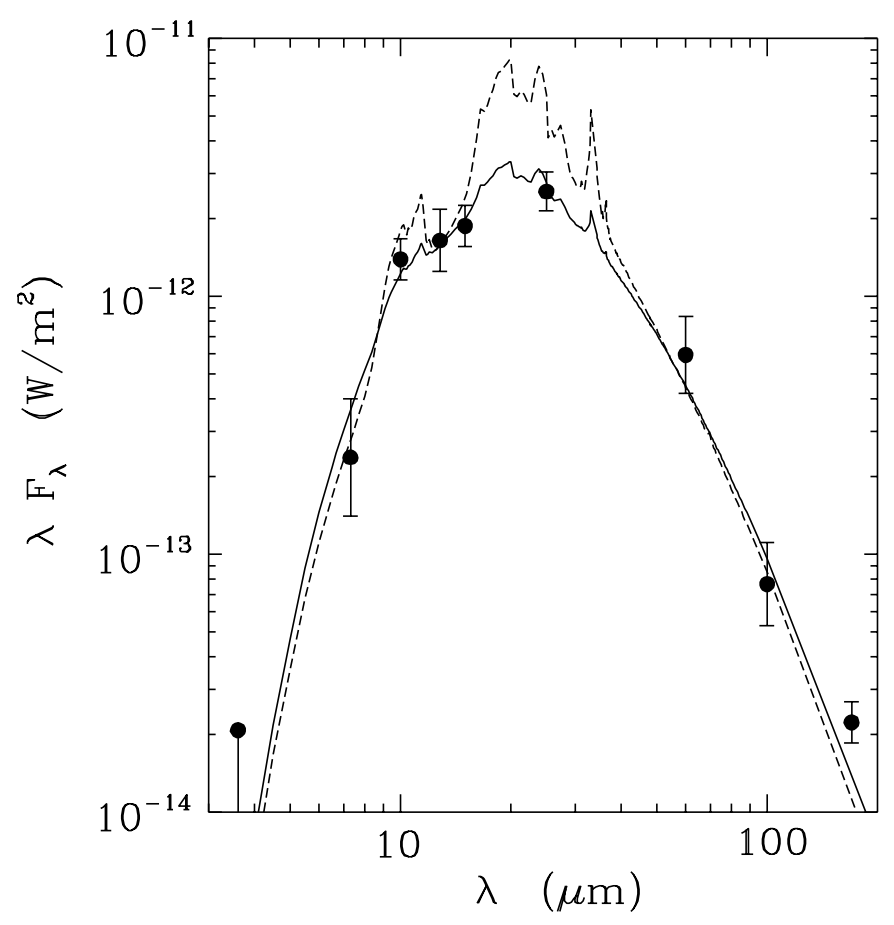

Fig. 8. Comparison of dust models with ISOPHOT data on 27 April 1996 at 4.6 AU. Dashed curve: same dust model as in Fig. 7, $N=$ 3.7. Solid curve: ratio of silicate to absorbing grains reduced by a factor of 3 .

in Figs. 6 (top panel) and 7. Clearly, the model predicts a far stronger silicate feature than is observed. The solid curve represents a model with the same size distribution, but the relative contribution of the silicate component is reduced by a factor of 3 . While this model does fit the data from $7.3-25 \mu \mathrm{m}$ within the error bars, the model is low at $10 \mu \mathrm{m}$ and too high at $20-25 \mu \mathrm{m}$. It is inconsistent with the strength of the $10 \mu \mathrm{m}$ silicate feature (ratio of the total $10 \mu \mathrm{m}$ flux to continuum a factor of $\sim 2$ ) in the 27 April PHT-S spectrum and ground-based spectra in June 1996 (Hayward \& Hanner 1997). No variation of model parameters using the same mixture of the various silicate minerals improved the fit at 10 and $25 \mu \mathrm{m}$. This suggests that the 20-25 $\mu \mathrm{m}$ silicate feature may have been weaker relative to the $10 \mu \mathrm{m}$ feature at $r \geq 4 \mathrm{AU}$. A ground-based spectrum taken at the UKIRT in June records a strong $10 \mu \mathrm{m}$ feature, but no discernable 20-25 $\mu \mathrm{m}$ feature, although the signal/noise was low (Davies et al. 1999). The answer cannot be simply a lower relative abundance of crystalline olivine in the coma in April, since the $11.3 \mu \mathrm{m}$ feature was strong. Unfortunately, the comet was too faint to acquire a full SWS spectrum in April to confirm the strength of the $20-25 \mu \mathrm{m}$ silicate feature.

The models presented here are representative of the dust properties fitting the observed SED. However, a full exploration of model parameters, including size distributions, grain mixtures, and porosity (e.g. Peschke 1997), is beyond the scope of this paper. For example, emission from icy grains may contribute to the long wavelength 
emission at 4.6 AU. Dust impact measurements during the GIOTTO Halley flyby yielded a mass distribution that did not follow a single power law, but was flatter at masses $>10^{-9} \mathrm{~kg}$. Li \& Greenberg (1998) have investigated a range of size distributions to fit Hale-Bopp IR data with a model in which the silicate grains have organic refractory mantles and the aggregate particles have a porosity $\sim 97 \%$.

\subsection{Icy grains}

At large heliocentric distances, where their sublimation rate is very low, water ice grains may be ejected during sublimation of $\mathrm{CO}$ or other volatile species. Ejection of water ice grains has been observed in the KOSI comet simulation experiments (Grün et al. 1993; Lämmerzahl et al. 1995). Broad absorption features of water ice at 1.5 and $2.05 \mu \mathrm{m}$ were detected in near-infrared spectra of Hale-Bopp at 7 AU (Davies et al. 1997).

Water ice has spectral bands near 44 and $65 \mu \mathrm{m}$. Excess emission at these wavelengths was detected in the ISO LWS spectrum of Hale-Bopp taken on 6 October at $r \sim 2.8$ AU by Lellouch et al. (1998). Their model predicted that $\sim 30 \%$ of the flux at $65 \mu \mathrm{m}$ was due to the emission from icy grains. One sees in Figs. 3d and 7 that the ISOPHOT $60 \mu \mathrm{m}$ flux on 7 October lies close to the continuum fit at shorter wavelengths. Thus, we cannot independently confirm the thermal emission from water ice grains at $2.8 \mathrm{AU}$. However, a $30 \%$ feature would be within the error bar on the $60 \mu \mathrm{m}$ flux.

At $2.8 \mathrm{AU}$, the blackbody temperature was $\sim 166 \mathrm{~K}$, high enough so that slightly dirty ice grains would be sublimating with an erosion rate $>10^{-8} \mathrm{~cm} / \mathrm{s}$ (Hanner 1981). Thus, we would expect icy grains to be more prominent in the coma beyond $4 \mathrm{AU}$ and we would anticipate a stronger emission feature in our earlier data. The $60 \mu \mathrm{m}$ flux/continuum ratio in our data on 27 April 1996 is $~ 1.7$, indeed higher than at $2.8 \mathrm{AU}$. The $60 \mu \mathrm{m}$ flux is also elevated in March.

Gaseous $\mathrm{OH}$ was first detected in Hale-Bopp in April 1996. Biver et al. (1997) derived a production rate $Q_{\mathrm{OH}} \sim 1.3 \times 10^{28} \mathrm{~mol} / \mathrm{s}$ from their $18 \mathrm{~cm}$ detection, corresponding to $Q_{\mathrm{H}_{2} \mathrm{O}} \sim 1.55 \times 10^{28} \mathrm{~mol} / \mathrm{s}$, while Weaver et al. (1997) derived $Q_{\mathrm{H}_{2} \mathrm{O}}=2 \times 10^{28} \mathrm{~mol} / \mathrm{s}$ at $4.79 \mathrm{AU}$ and $2.5 \times 10^{28} \mathrm{~mol} / \mathrm{s}$ at $4.3 \mathrm{AU}$ from HST and IUE UV spectra. The large $Q_{\mathrm{OH}} \sim 8 \times 10^{28} \mathrm{~mol} / \mathrm{s}$ derived by Schleicher et al. (1997) from optical spectroscopy may reflect uncertainties in the Haser model employed. For the present discussion, we take $Q_{\mathrm{H}_{2} \mathrm{O}} \sim 2 \times 10^{28} \mathrm{~mol} / \mathrm{s}$ at $4.6 \mathrm{AU}$.

The water sublimation rate, $Z(T)$, is a steep function of temperature, whereas the $60 \mu \mathrm{m}$ flux varies only slowly with temperature. Thus, the combination of the water production rate and the thermal emission allows us to set limits on the temperature and total cross section of the ice grains at $4.6 \mathrm{AU}$, a technique previously applied to comet Bowell (Hanner \& Campins 1986).
The surface area required to generate the observed $\mathrm{OH}$ via sublimation if the grains were at the blackbody temperature of $130 \mathrm{~K}$ is $\sim 4 \times 10^{13} \mathrm{~m}^{2}$. This surface area would give rise to observable $60 \mu \mathrm{m}$ thermal emission of $\sim 8 \times 10^{-13} \mathrm{~W} / \mathrm{m}^{2} / \mu \mathrm{m}$. The size of the coma reported by visual observers in April was $\sim 10^{\prime}$ (Green 1996) and the coma filled the $14^{\prime}$ field of view of the ground-based $R$ images on 27 April (Table 2). If the diameter of the coma was 15 arcmin and if icy grains were distributed within this volume $\propto 1 / \rho$, then $\sim 3 \%$ of the emission would be within the $23^{\prime \prime}$ ISOPHOT field of view, or $\sim 2 \times 10^{-14} \mathrm{~W} / \mathrm{m}^{2} / \mu \mathrm{m}$, a factor of 2 higher than our total observed $60 \mu \mathrm{m}$ flux.

If the ice grain temperature were even a few degrees colder, $T=125 \mathrm{~K}$, then the required sublimation area would be $\sim 3 \times 10^{14} \mathrm{~m}^{2}$, producing a $60 \mu \mathrm{m}$ flux of $\sim 1.5 \times 10^{-13} \mathrm{~W} / \mathrm{m}^{2} / \mu \mathrm{m}$ within a $23^{\prime \prime}$ aperture, 15 times the total observed flux. Thus, the blackbody temperature of $130 \mathrm{~K}$ is effectively a lower limit to the temperature of the sublimating ice grains. A temperature of $136 \mathrm{~K}$ would correspond to a sublimating area of $\sim 5 \times 10^{12} \mathrm{~m}^{2}$ and a thermal flux within our $23^{\prime \prime}$ aperture of $\sim 3 \times 10^{-15} \mathrm{~W} / \mathrm{m}^{2} / \mu \mathrm{m}$, more consistent with the observations. If, on the other hand, the ice grain temperature were as high as $142 \mathrm{~K}$, then the ice grains contributing to the observed $60 \mu \mathrm{m}$ excess flux in the original $135^{\prime \prime} \times 135^{\prime \prime}$ field of view would sublimate $\sim 2 \times 10^{28} \mathrm{~mol} / \mathrm{s}$, comparable to the total observed $Q_{\mathrm{H}_{2} \mathrm{O}}$ from the entire coma. This sets an effective upper limit on the temperature of the ice grains at $4.6 \mathrm{AU}$.

We conclude that the sublimating ice grains in the coma at 4.6 AU must have been a few $\mathrm{K}$ warmer than the blackbody temperature of $130 \mathrm{~K}$, in order to balance the observed thermal emission and water production rate. While pure water ice grains absorb very little solar radiation and can remain very cold, Hanner (1981) showed that even a slight admixture of absorbing material drastically lowers the albedo and increases the grain temperature. At large $r$, where the energy going into sublimation is low, slightly dirty ice grains can reach or exceed the blackbody temperature. Mukai \& Mukai (1984) calculated that $100 \mu \mathrm{m}$ radius grains with a volume fraction of small absorbing particles $\geq 0.1$ will reach $T \sim 140 \mathrm{~K}$ at $4.5 \mathrm{AU}$.

The total icy grain surface area of $\sim 5 \times 10^{12} \mathrm{~m}^{2}$ at $T=$ $136 \mathrm{~K}$ would correspond to a total mass of $\sim 2 \times 10^{10} \mathrm{~kg}$ if the grain radius was $\sim 15 \mu \mathrm{m}$, as estimated by Lellouch et al. (1998) and $\sim 2 \times 10^{11} \mathrm{~kg}$ if the grain radius was $\sim 100 \mu \mathrm{m}$.

Amorphous water ice does not have a spectral feature at $65 \mu \mathrm{m}$; thus, the presence of excess emission near $65 \mu \mathrm{m}$ indicates that the emitting grains were crystalline. Water ice undergoes a phase change from amorphous to cubic at $T \sim 140 \mathrm{~K}$. It is possible that the phase change could have taken place in the coma at $4.6 \mathrm{AU}$, given the grain temperatures we have derived above. If so, the exothermic phase transition would have contributed energy for sublimation. However, the $60 \mu \mathrm{m}$ flux was also high in our March data at $4.9 \mathrm{AU}$, when the grains would have been several degrees cooler. Thus, we cannot say conclusively whether 
Table 9. Hale-Bopp dust production rates.

\begin{tabular}{lrrr}
\hline$R(\mathrm{AU})$ & \multicolumn{2}{c}{$Q_{\mathrm{d}}(\mathrm{kg} / \mathrm{s})$} & $\psi$ \\
& $N=3.7$ & $N=3.5$ & \\
\hline 4.58 & $1.5 \times 10^{4}$ & $3.0 \times 10^{4}$ & 9 \\
2.82 & $7.0 \times 10^{4}$ & $1.5 \times 10^{5}$ & 6 \\
3.90 & $2.0 \times 10^{4}$ & $5.0 \times 10^{4}$ & 10 \\
\hline
\end{tabular}

the ice was in crystalline form before ejection from the nucleus.

\subsection{Dust production}

Dust production rates can be computed from the model fits to the observed fluxes, following the method outlined in Hanner (1984). We have

$Q_{\mathrm{d}}=M_{\mathrm{d}} \frac{2 \bar{v}}{\pi \rho_{0}}$

where $\bar{v}$ is the mean mass-weighted dust particle velocity, integrated over the size distribution, $\rho_{0}$ is the projected aperture radius, and $M_{\mathrm{d}}=F_{\mathrm{obs}} \Delta^{2} F_{\mathrm{c}}$ is the mass of dust within the field of view. The quantity $F_{\mathrm{c}}$ is the emission/gm computed from the dust models described above. A semi-empirical expression for $v(a)$ was applied (Sekanina 1981), calibrated to Hale-Bopp using the coefficients a and b from (Vasundhara \& Chakraborty 1999).

The dust production rates are presented in Table 9 . We assumed a grain density of $1 \mathrm{~g} / \mathrm{cm}^{3}$ and a maximum grain radius of $1 \mathrm{~cm}$. Results for both $N=3.7$ and $N=3.5$ in the power-law size distribution are shown for comparison; however, the $Q_{\mathrm{d}}$ for $N=3.5$ are the appropriate values to use. The uncertainty due to $\bar{v}$ and grain density is at least a factor of two, particularly the heliocentric dependence of $\bar{v}$. The mass loss in the form of large particles, $a \sim 1 \mathrm{~cm}$ or larger, is an additional uncertainty.

The dust/gas mass ratio, $\psi$, is based on $Q_{\mathrm{d}}$ for $N=3.5$ and the gas production rates in (Biver et al. 1999). The relatively high mass loading is consistent with values inferred by other investigators. Jewitt \& Matthews (1999) derived $Q_{\mathrm{d}} \sim 2 \times 10^{6} \mathrm{~kg} / \mathrm{s}$ near 1 AU from submm continuum observations, which are sensitive to large particles, leading to $\psi \sim 6$. Lisse et al. (1999) find $\psi \sim 5$ on 31 October 1996. Vasundhara \& Chakraborty (1999) find $\psi \sim 3-6$ in 1997. The high mass loading at large $r$, when CO dominated the gas production, is particularly interesting. Similarly high values were inferred by Sekanina (1996) when the comet was at 6 AU preperihelion.

\section{Conclusions}

Comet Hale-Bopp was an excellent target-of-opportunity object for ISO. Five sets of broad bandpass photometric measurements were taken with the ISOPHOT photometer at heliocentric distances, $r=4.9,4.6,2.9,2.8$ AU pre-perihelion and 3.9 AU post-perihelion. Each data set provided the spectral energy distribution (SED) from
3.6-170 $\mu \mathrm{m}$. Observing times were selected to minimize the astronomical background and measurements of the sky background were performed with identical instrument settings at the same sky positions several days after the comet's passage.

We have described in detail the data reduction procedure and assessment of measurement uncertainties, including our techniques to correct for variable detector responsivity using the Fine Calibration Source. The final fluxes have been corrected for sky background, PSF, and positional offsets and have been reduced to a common aperture size, assuming a $1 / \rho$ brightness distribution. The final fluxes are in reasonable agreement, within the uncertainties, with PHT-S, SWS, and LWS spectra.

The resulting SED for each data set allows us to draw some general conclusions about the properties of the grains. Planck functions fitted to the continuum data points at 7.3 and $12.8 \mu \mathrm{m}$ yield color temperatures a factor of $\sim 1.5$ above the corresponding blackbody temperature throughout the apparition. These are the highest color temperatures, relative to $T_{\mathrm{bb}}$, yet observed for a comet. While the color temperature is not equal to the physical temperature of the grains, the high values do indicate a substantial population of small, warm absorbing grains in the coma, even at large heliocentric distances. However, the SED did not decrease steeply between 12.8 and $170 \mu \mathrm{m}$, as would be the case if only small grains were present.

The total emitted energy varied $\propto r^{-3}$, reflecting the strong activity due to $\mathrm{CO}$ at large heliocentric distance. If the dust production rate scaled directly with insolation, one would expect a $r^{-4}$ dependence; comets typically follow a $r^{-4}$ dependence at smaller $r$ (Gehrz \& Ney 1992). Hale-Bopp maintained a high dust production even at 3.9 AU post-perihelion; the total thermal energy on December 30, 1997 falls close to the relation defined by the preperihelion values.

Strong excess emission at 10 and $25 \mu \mathrm{m}$, attributed to small silicate grains, was evident in all our data sets, even at 4.6-4.9 AU, the largest heliocentric distance that silicate emission has been detected in a comet. The feature/continuum ratio increased as the comet approached the sun.

Simple two-component models for a mixture of submicron silicate grains, including both glassy and crystalline silicates, and a broad size distribution of featureless absorbing particles were fit to the spectra and photometry at 2.8 AU pre-perihelion. Matching the slope of the observed SED out to $\lambda>100 \mu \mathrm{m}$ requires a size distribution in which most of the mass in the coma resides in large particles. The models require a factor of $\sim 3$ higher crosssection of small silicate grains relative to featureless grains at 2.8 AU than at 4.6 AU in order to fit the 10 and $25 \mu \mathrm{m}$ emission. Production rates derived from the dust models imply a high dust mass loading of the outflowing gas, even at large heliocentric distances.

The albedo, $A(\theta)=0.26-0.28$, was computed from ground-based $R$-band fluxes and the ISOPHOT thermal 
emission at 4.8 and $2.8 \mathrm{AU}$ (phase angle $12^{\circ}$ and $19^{\circ}$ ). This albedo is higher than that of comet Halley $(A(\theta) \sim$ 0.2 ; Gehrz \& Ney 1992) and other comets and at the low end of the range found for Hale-Bopp at smaller $r$ (Mason et al. 2000). There is no evidence for a component of cold, bright icy grains enhancing the scattered light at $4.6 \mathrm{AU}$.

However, there is possible excess emission from crystalline water ice grains at $60 \mu \mathrm{m}$ in the ISOPHOT data at 4.6-4.9 AU preperihelion. The $60 \mu \mathrm{m}$ flux and the observed $\mathrm{OH}$ production rate at $4.6 \mathrm{AU}$ are consistent if the temperature of the sublimating water ice grains was $\sim 136 \mathrm{~K}$, slightly warmer than an equilibrium blackbody and close to the temperature of the phase change between amorphous and crystalline ice ( $140 \mathrm{~K})$. Thus, we cannot say conclusively whether the ice was in crystalline form before ejection from the nucleus. The total surface area of sublimating grains necessary to supply the observed $Q_{\mathrm{OH}}$ at $136 \mathrm{~K}$ is $\sim 5 \times 10^{12} \mathrm{~m}^{2}$, corresponding to a mass of $\sim 2 \times 10^{10} \mathrm{~kg}(15 \mu \mathrm{m}$ radius grains $)$ to $\sim 2 \times 10^{11} \mathrm{~kg}$ (100 $\mu$ m radius grains).

In summary, comet Hale-Bopp displayed a wide variety of interesting phenomena, well worth the investment of ISO resources. In particular, the IR observations at 4.6$4.9 \mathrm{AU}$ are unique in displaying the dust properties in a comet at large heliocentric distance, where $\mathrm{CO}$ dominated the sublimation. The strong thermal emission from grains warmer than a blackbody and the strong $10 \mu \mathrm{m}$ silicate emission feature were unexpected at this large heliocentric distance, where one might have expected small refractory grains to remain embedded in water ice. We await the study of other comets at large heliocentric distances from future infrared space missions.

Acknowledgements. Observations were obtained with ISO, an ESA project, with instruments funded by ESA Member States (especially the PI countries: France, Germany, The Netherlands and the UK) and with the participation of ISAS and NASA. We acknowledge the support of Dr. Heike Rauer, DLR Berlin, Dr. Rita Schulz, ESTEC Noordwijk, and Dr. Gian-Paolo Tozzi, Arcetri Observatory Firenze, in acquiring the ground-based observations of the comet at ESO La Silla. Support by the DLR for S. Peschke is acknowledged. M. Hanner's research was carried out at the Jet Propulsion Laboratory, California Institute of Technology, under contract with NASA. H. Campins acknowledges the support of NASA and NSF. C. Lisse acknowledges the support of NASA.

\section{Appendix A: Signal processing}

The whole data set was reduced homogeneously with the ISOPHOT interactive analysis package (PIA) version 7.1 (Gabriel et al. 1997, 1998; Gabriel \& Acosta-Pulido 1999). The essential data processing steps are described in the PIA user manual (http://www.iso.vilspa.esa.es/manuals / PHT / pia/ um/pia_um.html, chapter 2). The signals per integration ramp were determined by first order fits to each ramps (method 1) as well as by pair wise differences of subsequent read-outs (method 2). Further signal processing was identical for both methods. Independently, pair wise differences for all read-outs of a measurement were computed after applying the non-linearity correction on ERD level. The signal was derived by applying the "sample myriad" (Kalluri \& Arce 1998) to the pair wise differences and adopting the mode of the histogram of the distribution as signal (method 3). Signal calibration was always performed in PIA, independent of how the signal was derived. Given the wide flux range of the observations and the peculiarities of the detectors various extra considerations had to be added to the signal derivation process:

1. Digitization noise. In case there is sufficient signal, the best results are obtained with the myriad method (Table 3, proc.: M). However, in cases of very low fluxes the number of digitization steps per read-out is small and consequently digitization noise is significant. The mean number of digitization steps per subsequent readouts, nstep, was calculated for object and FCS:

$$
\frac{\text { nstep }=S C P \times I N T T}{N D R \times A D C}
$$

where $S C P$ is the signal $(\mathrm{V} / \mathrm{s})$ on $S C P$ level, $I N T T$ is integration time per ramp (s), NDR is the number of non-destructive readouts per ramp, and $A D C=$ $610 \mu \mathrm{V}$ is the digitization accuracy. In case nstep $<5$, we use a first order fit to the full ramp (Table 3, proc.: F) in order to derive the signal and to calculate the flux-dependent nominal responsivity;

2. Dark current. The standard dark current subtraction was applied in order to arrive at the object signal, $S$. For most data sets the object signal exceeded the dark current signals, $D$, by far. However several of the $3.6 \mu \mathrm{m}$ measurements had $S / D<1$. In these cases, no reliable signal and responsivity values could be obtained and we had to resort to the default responsivity value (Table 3, type: d). We set the signal value to the dark current value $D$ (provided by PIA; Table 3, proc.: D) and used this value as an upper limit (Table 3, uncert.: u.l.).

\section{Appendix B: Responsivity determination}

It is known that the detector responsivity varies, for a number of reasons (orbit position of ISO, previous and current exposure to infrared radiation). Therefore, the actual responsivity at the time of observation must be determined by an FCS measurement. We planned our Hale-Bopp observations so that each individual comet observation was followed by an FCS measurement using the same filter and the same aperture. The most important variation of the detector responsivity is due to the infrared flux level itself: at high flux levels the responsivity is generally low. 
Therefore, the FCS measurement provides a relevant responsivity only if its signal is comparable (within a factor of 3) to the object signal. Since the FCS settings were preplanned on the basis of expected fluxes long before the observations were taken, large deviations between FCS and object signals sometimes occured.

In the initial data reduction described by Peschke (1997) and Grün et al. (1999), the default responsivity (a fixed value for each detector and filter) was used in case of such deviations. Its uncertainty was defined as the range of responsivities calculated by the different processing methods, including the default responsivity. This procedure resulted in large uncertainties of up to a factor of three. In the present analysis, we apply an improved data reduction scheme that is based on the flux dependence of the detector responsivities derived by the ISOPHOT calibration team (ISOPHOT Off-line Processing Report, Klaas 1999). For this nominal responsivity, one assumes that it depends only on the flux received by the detector. In the data processing described here, we correct the actual responsivities obtained from FCS measurements for the different object flux level (for the definition of the corrected responsivity see item 3 , below) and determine the uncertainty from the residual deviation of our corrected responsivity from the nominal flux-dependent responsivity. In this way, we obtain improved inband power values and smaller, but still realistic, uncertainties. The mean uncertainty of all measurements that returned a positive signal is $25 \%$. Table 3 displays a summary of the observational parameters that were considered and the result of the initial data processing.

The following considerations were applied in order to arrive at reliable detector responsivities:

1. Only 4 readouts per ramp and saturation effects. In case of only 4 readouts per ramp, only the two middle data points are left for the slope determination of the ramp, after applying the standard PIA (de)selection criteria. If, in addition, the FCS signal reached saturation (Table 3, remarks: so, sF) we have no confidence in the obtained actual responsivity and use the signaldependent nominal responsivity, $R_{\text {nom }}$ (Klaas 1999, Table 3, type: $\mathrm{n}$ ), instead. As measure of the uncertainty, we still use the deviation of the nominal from the actual responsivity.

2. FCS heating power out of limits. In some cases the FCS heating power was outside the recommended range (Table 3, remarks: w1 = heating power below, and w2 $=$ heating power above recommended range). Except for $12.8 \mu \mathrm{m}$, the deviations from the allowed range were significant and we do not trust the responsivities derived from the FCS. We use default responsivities (Table 3, type: d) when the heating power was below the allowed range and the nominal responsivity (Table 3, type: n) when it was above the allowed range. As the uncertainty, we use the deviation of the default responsivity from the nominal responsivity.
3. Ratio of object and FCS signals and calculation of the corrected responsivity. The ratio of object and FCS signals $\left(r_{\mathrm{SCP}}\right)$ is an indicator of how well the FCS derived calibration relates to the object measurement. If the signal measured from the object and that from the corresponding FCS measurement are of similar level, good calibration results and the actual responsivity, $R_{\text {act }}$ obtained can be used reliably to calculate the inband power value from the signal height. In case the object and FCS signals differ by more than a factor of three (Table 3 remarks: r3), significant deviations of the inband power calibration may result. In the previous data reduction (Peschke 1997; Grün et al. 1999), the default responsivity was used in this case; thereby large uncertainties (deviations between actual and default responsivity of up to factor 3 ) were the consequence. Different from that procedure, now we have corrected the actual responsivities determined from our FCS measurements even in the case of large object to FCS ratios. We first determined from the object and FCS signals the corresponding nominal responsivities ( $R_{\text {nom }}$ Object, $R_{\text {nom }}$ FCS $)$ from tables provided by the ISOPHOT team (Klaas 1999). In a second step we scaled the actual responsivity by the ratio of the nominal responsivities and obtained the corrected responsivity, $R$ (Table 3, type: c):

$$
R=R_{\text {act }} \frac{R_{\text {nom }} \text { Object }}{R_{\text {nom }} \text { FCS }}
$$

\section{Appendix C: Inband power calculation}

The inband power in watts (Table 3 , signal) was calculated by PIA from the signal $S$ in $\mathrm{V} / \mathrm{s}$, using the responsivity $R(\mathrm{~A} / \mathrm{W})$ from Table 3 (respons):

$P=\frac{S \times C_{\mathrm{int}}}{R}$

with the integration capacity, $C_{\text {int }}=9 \times 10^{-14} F$ for all but the $\mathrm{C} 2$ detector $\left(14 \times 10^{-14} F\right)$. For the $\mathrm{C}$ detectors, inband powers of all pixels were summed. At this stage of processing, we consider only the total inband power of all pixels of the $\mathrm{C} 1$ and $\mathrm{C} 2$ detectors. Variations of the brightnesses recorded by individual pixels of the flat-fielded Hale-Bopp observations reached factors up to 25 due to brightness variations in the coma, whereas pixel variations of the shadow observations were typically within a factor of 2 .

\section{Appendix D: Inband power uncertainties}

There are two different independent contributions to the uncertainty (Table 3, uncert.): the statistical error of the measurement, which is typically of the order of one to a few percent, and the difference between responsivities determined by the two methods (biggest contribution to the uncertainty). We used as the uncertainty of the responsivities the deviation of the corrected responsivity from the best fit to the flux-dependent responsivity. If this deviation was smaller than the $(1-\sigma)$ uncertainty of the FCS 
calibration stated by the ISOPHOT team, we used the latter. Responsivity uncertainties for C- detectors were averaged over all pixels. In case the signal was smaller than the dark current, we set the signal value to the dark current value and used this value as an upper limit. The mean uncertainty of all observations is $25 \%$, caused mainly by a combination of pointing offsets, varying detector responsivity, and general difficulties to calibrate extended sources.

In the future, improved inband power values may be obtained when we succeed in applying the myriad, or any other enhanced method, also to small signal levels. Improved values and reduced uncertainties may result also from a better understanding of the relation of our FCSderived responsivities to the flux-dependent responsivities and the uncertainties involved with both.

Another, so far not well determined, contribution to the uncertainty arises from the problem that the flux of extended sources is calculated with a calibration procedure based on point sources only. First investigations of a point source placed at different positions within the aperture showed that the sensitivity varies significantly with the position of that source (Müller 2000a, 2000b). This affects mainly bright sources far away from the center of the aperture and extended sources with bright structures at the edge of the aperture.

\section{References}

A'Hearn, M. F., Schleicher, D. G., Feldman, P. D., Millis, R. L., \& Thompson, D. T. 1984, AJ, 89, 579

Biver, N., Bockelée-Morvan, D., Colom, P., et al. 1997, Science, 275, 1915

Biver, N., Rauer, H., Despois, D., et al. 1996, Nature, 380, 137

Boehnhardt, H., Birkle, K., Fiedler, A., et al. 1999, Earth, Moon \& Planets, 78, 179

Clegg, P. E., Ade, P. A. R., Armand, C., et al. 1996, A\&A, 315, L38

Colom, P., Gérard, E., Crovisier, J., et al. 1999, Earth, Moon \& Planets, 78, 37

Crovisier, J., Brooke, T. Y., Hanner, M. S., et al. 1996, A\&A, 315, L385

Crovisier, J., Brooke, T. Y., Leech, K., et al. 2000, in Thermal Emission Spectroscopy and Analysis of Dust Disks and Regoliths, ed. M. L. Sitko, A. L. Sprague, \& D. K. Lynch, ASP Conf. Ser., 196, 109

Crovisier, J., Leech, K., Bockelée-Morvan, D., et al. 1997a, Science, 275, 1904

Crovisier, J., Leech, K., Bockelée-Morvan, D., et al. 1997b, in Proc. First ISO Workshop on Analytical Spectroscopy, ESA SP-419, 137

Crovisier, J., Leech, K., Bockelée-Morvan, D., et al. 1999, in The Universe as seen by ISO, ESA SP-427, 137

Davies, J. K., Geballe, T. R., Hanner, M. S., et al. 1999, Earth, Moon \& Planets, 78, 293

Davies, J. K., Roush, T. L., Cruikshank, D. P., et al. 1997, Icarus, 127, 238

Dorschner, J., Begemann, B., Henning, Th., Jäger, C., \& Mutschke, H. 1995, A\&A, 300, 503

Edoh, O. 1983, Ph.D. Thesis, University of Arizona
Fernandez, Y. R., Wellnitz, D. D., Buie, M. W., et al. 1999, Icarus, 140, 205

Gabriel, C., et al. 1997, The ISOPHOT Interactive Analysis PIA, a calibration and scientific analysis tool, in ASP Conf. Ser., 125, Astronomical Data Analysis Software and Systems VI, ed. G. Hunt, H. E. Payne (San Francisco: ASP), 108

Gabriel, C., Acosta-Pulido, J., \& Heinrichsen, I. 1998, News on the ISOPHOT Interactive Analysis PIA, in ASP Conf. Ser., 145, Astronomical Data Analysis Software and Systems VII, ed. R. Albrecht, R. N. Hook, \& H. A. Bushouse (San Francisco), 165

Gabriel, C., Acosta-Pulido, J. A. 1999, From a calibration tool to a full scientific analysis and pipeline system: the ISOPHOT Interactive Analysis (PIA), in ESA SP-427, The Universe as Seen by ISO, ed. P. Cox, \& M. F. Kessler, 73

Gehrz, R. D., \& Ney, E. P. 1992, Icarus, 100, 162

Green, D. W. E. 1996, IAU Circ, No. 6401

Grün, E., Gebhard, J., Bar-Nun, A., et al. 1993, JGR, 98, 15091

Grün, E., Peschke, S. B., Stickel, M., et al. 1999, in ESA SP-427, The Universe as Seen by ISO, ed. P. Cox, \& M. F. Kessler, 181

Hanner, M. S. 1981, Icarus, 47, 342

Hanner, M. S. 1983, in Cometary Exploration, ed. T. I. Gombosi (Hungar. Acad. Sci., Budapest), II, 1

Hanner, M. S., \& Campins, H. 1986, Icarus, 67, 51

Hanner, M. S., Giese, R. H., Weiss, K., \& Zerull, R. 1981, A\&A, 104,42

Hayward, T. L., Hanner, M. S., \& Sekanina, Z. 2000, ApJ, 538, 428

Hayward, T. L., \& Hanner, M. S. 1997, Science, 275, 1907

ISSA 1994, IRAS All-Sky Survey, IPAC, JPL/Caltec, Pasadena IRSKY, An Observation Planning Tool for the Infrared Sky 1995, IRAS All-Sky Survey, IPAC, JPL/Caltech, Pasadena Jewitt, D., \& Matthews, H. 1999, AJ, 117, 1056

Jewitt, D., Senay, M., \& Matthews, H. 1996, Science, 271, 1110 Jorda, L., Lamy, P., Groussin, O., A'Hearn, M. F., \& Peschke, S. 2000, ISO Beyond Point Sources: studies of extended infrared emission, Proceedings, ESA SP-455

Jorda, L., Lamy, P. L., Groussin, O., et al. 2000, ISOCAM observations of cometary nuclei, in Proc. Workshop ISO beyond point sources, ESA SP, in press

Kalluri, S., \& Arce, G. R. 1998, IEEE Trans. Sig. Proc. 46, 322

Kessler, M. F., Steinz, J. A., Anderegg, M. E., et al. 1996, A\&A, 315, L27

Kissel, J., et al. 1986, Nature, 321, 280, 336

Klaas, U. 1999, ISOPHOT Off-line Processing Report

Klaas, U., Krüger, H., Heinrichsen, I., \& Laureijs, R. 1994, ISOPHOT Observers Manual, ISO Science Operations Team, Noordwijk, ESA/ESTEC

Koike, C., Shibai, H., \& Tuchiyama, A. 1993, MNRAS, 264, 654

Lämmerzahl, P., Gebhard, J., Grün, E., \& Klees, G. 1995, Plan. Space Sci., 43, 363

Lamy, P., Jorda, L., Toth, I., et al. 1999, BAAS, 31, 1116

Landolt, A. U. 1992, AJ, 104, 340

Lellouch, E., Crovisier, J., Lim, T., et al. 1998, A\&A, 339, L9

Lemke, D., Klaas, U., Abolins, J., et al. 1996, A\&A, 315, L64

Lena, P., et al. 1998, Observational Astrophysics (Springer Press, Heidelberg)

Li, A., \& Greenberg, J. M. 1998, ApJL, 498, L83

Lisse, C. M., A'Hearn, M. F., Hauser, M. G., et al. 1998, ApJ, 496, 971 
Lisse, C. M., Fernandez, Y. R., A'Hearn, M. F., et al. 1999, Earth, Moon, and Planets, 78, 251

Mason, C. G., Gehrz, R. D., Jones, T. J., et al. 2000, ApJ, in press

Millis, R. L., A'Hearn, M. F., \& Thompson, D. T. 1982, AJ, 87,1310

Müller, T. G. 2000a, ISOPHOT Aperture Profiles and Background Measurements, ISO beyond point sources: studies of extended infrared emission, Proceedings, ESA SP-455

Müller, T. G. 2000b, ISOPHOT Aperture Sequences on Pointand Extended Sources, ISO beyond point sources: studies of extended infrared emission, Proceedings, ESA SP-455

Mukai, T., \& Mukai, S. 1984, Adv. Space Res. 4(9), 207

Ney, E. P. 1982, in Comets, ed. L. L. Wilkening (Univ. Arizona, Tucson), 323

Peschke, S. B. 1997, Ph.D. Thesis, University of Heidelberg

Peschke, S. B., Stickel, M., Heinrichsen, I., et al. 1998, First maps of comet Hale-Bopp in the far-infrared, in relation to radial profiles at other wavelengths, in ESA SP-427, The Universe as Seen by ISO, ed. P. Cox, \& M. F. Kessler, 185

Schleicher, D. G., Lederer, S. M., Millis, R. L., \& Farnham, T. L. 1997, Science 275, 1913
Sekanina, Z. 1981, in The Comet Halley Dust and Gas Environment, ed. B. Battrick \& E. Swallow, ESA SP-174, ESTEC, Noordwijk, The Netherlands, 55

Sekanina, Z. 1996, A\&A, 314, 957

Sekanina, Z. 1999, Earth, Moon \& Planets, 77, 147

Tokunaga, A. T., Golisch, W. F., Griep, D. M. Kaminski, C. D., \& Hanner, M. S. 1986, AJ, 92, 1183

Tokunaga, A. T., Golisch, W. F., Griep, D. M., Kaminski, C. D., \& Hanner, M. S. 1988, AJ, 96, 1971

van de Hulst, H. C. 1957, Light Scattering by Small Particles (Wiley, New York)

Vasundhara, R., \& Chakraborty, P. 1999, Icarus, 140, 221.

Weaver, H. A., Brooke, T. Y., Chin, G., et al. 1999, Earth, Moon \& Planets, 78, 71

Weaver, H. A., Feldman, P. D., A'Hearn, M. F., et al. 1997, Science, 275, 1900

Weaver, H. A., \& Lamy, P. 1999, Earth, Moon \& Planets, 79, 17

Williams, D. M., Mason, C. G., \& Gehrz, R. D., et al. 1997, ApJ, 489, L91 